\title{
A UNITARY REPRESENTATION OF THE CONFORMAL GROUP ON MINKOWSKI SPACE AND DYNAMICAL GROUPS I
}

\section{ERNEST THIELEKER}

This paper studies the so-called dynamical group of the $n$-dimensional non-relativistic quantum mechanical Kepler problem. This group turns out to be isomorphic to the real pseudo-orthogonal group $\mathrm{O}(2, n+1)$. First it is shown that there exists a Lie algebra $G$ of formal differential operators on $\mathbb{R}^{n}$ which has the following properties: The algebra $G$ is isomorphic to the Lie algebra $\operatorname{so}(2, n+1)$ and contains the formal hamiltonian operators for the positive and negative energy spectra of the Kepler problem. This much is done in the spirit of the work in the physics literature for the case $n=3$. The main results of the paper show that there exists a unitary representation of the group $\mathrm{O}(2, n+1)$ whose differential is a skew self adjoint extension of the Lie algebra $G$. In outline, this group representation arises as a certain intregral transform of a solution space of the $(n+1)$-dimensional wave equation on $\mathbb{R}^{n+1}$. The latter solution space is derived from a suitable non-unitary induced representation of $\mathrm{O}(2, n+1)$ induced by a certain maximal parabolic subgroup.

1. Introduction. Let $n$ be a positive integer and let for $y \in \mathbb{R}^{n}$, Euclidean real $n$-space, $r(y)=\sqrt{y_{1}^{2}+\cdots+y_{n}^{2}}$. Write $\partial_{i}=\partial / \partial y_{i}$ for $1 \leq i \leq n$. Let $\Delta$ be the Laplace operator $\Delta=\partial_{1}^{2}+\cdots+\partial_{n}^{2}$ on $\mathbb{R}^{n}$. In suitable units the Schrödinger equation for the "hydrogen atom" (ignoring fine structure) is

$$
\left(z / r-\frac{1}{2} \Delta\right) \Psi(y)=\lambda \Psi(y),
$$

where $z$ is a parameter. The eigenfunctions $\Psi$ belong to a suitable linear space of differentiable functions. This equation is "obviously" symmetric under the orthogonal group on $\mathbb{R}^{n}$. In other words, the commutant of the operator $H:=\left(z / r-\frac{1}{2} \Delta\right)$ contains the operators representing the rotations on $\mathbb{R}^{n}$. Taking the infinitesimal point of view, this says that the commutant of this operator contains the skew adjoint operators which are the infinitesimal generators of the rotations. Now this would also be the case if the Coulomb potential $-z / r$ were replaced by "any" function $V: \mathbb{R}^{n} \Rightarrow \mathbb{R}$ of the form $V(y)=W(r)$. 
However it was pointed out by Fock [F] and Bargmann [B1] for the case $n=3$ and Alliluev for general $n$, that because of the special form of the Coulomb potential, the equation (1) possesses a higher symmetry: In addition to the infinitesimal generators of the rotations, the commutant of $H$ also contains differential operators of the second order, which are formally skew adjoint. These together with the infinitesimal rotations generate a Lie algebra isomorphic to the Lie algebra of a (pseudo-)orthogonal group of $\mathbb{R}^{n+1}$. For negative elements of the spectrum this is the group $\mathrm{SO}(n+1)$, and for the positive spectrum this group is $\mathrm{SO}(n, 1)$. An important consequence of these results is that the eigenspace of operator $H$ are representation modules for these higher dimensional orthogonal groups. This gives rise to the so-called accidental degeneracy of the spectrum of $H$.

However an even larger finite-dimensional Lie algebra comes into play here. By making a change of variables $y_{i} \Rightarrow \sqrt{ \pm 2} \lambda y_{i}, i=1, \ldots, n$, (the + sign for the positive eigenvalues, and - sign for the negative eigenvalues) one can rewrite (1.1) as two equations:

$$
\begin{aligned}
& \{r \Delta-r\} \psi=z_{-} \psi, \\
& \{r \Delta+r\} \psi=z_{+} \psi,
\end{aligned}
$$

where $z_{ \pm}=2 z / \sqrt{ \pm 2 \lambda}$. The first equation is equivalent to (1.1) for negative eigenvalues, while the second equation is equivalent to (1.1) for positive eigenvalues. One then replaces the original problem by another related problem: One considers the operators on the left hand sides of equations $\left(1.2_{ \pm}\right)$as acting on the same function space, and considers the eigenfunctions and eigenvalues of both operators. This problem differs from the original one. In fact, if orthogonal projection is defined relative to usual $L_{2}$ Hilbert space structure, the projections of the original Schrödinger operator corresponding to the positive and the negative spectra commute with each other, while the operators $r-r \Delta$ and $r+r \Delta$ do not commute. However it turns out that the spectra and spectral multiplicity of each of these operators are the same as the operators derived from the orthogonal projections. Now in the case $n=3$ it was pointed out in Barut [Ba], that the operators $i(r-r \Delta)$, and $i(r+r \Delta)$ generate a Lie algebra isomorphic to the Lie algebra $\operatorname{sl}(2, \mathbb{R})$. Moreover, what is more remarkable, if one tosses in the first and second order differential operators which either commute with $r-r \Delta$ or with $r+r \Delta$, the Lie algebra that is generated by such operators remains finite-dimensional and is isomorphic to so $(2, n+1)$. 
The generalization to arbitrary $n$ can be obtained by methods similar to those of Barut. (See §2.) In case $n=3$, this Lie algebra is generally called the dynamical Lie algebra of the system. We apply this terminology to the case of general $n$.

A main result of this paper is to prove the existence of a unitary representation of the group $\mathrm{O}(2, n+1)$ that gives rise to the above dynamical Lie algebras of operators as infinitesimal generators of the one parameter subgroups in this representation. The irreducibility of this representation is taken up in the next paper in this series. Later we intend to study various classes of representations of the groups $\mathrm{SO}(2, n+1)$ and their spinor covering groups which generalize this representation. Along the way we develop some geometric results on Möbius transformations on the Minkowski spaces. The paper also contains a result expressing the class one spherical functions of the generalized Lorentz group $\mathrm{SO}_{e}(1, n)$ in terms of solutions of the wave equation on $(n+1)$-dimensional space (Proposition 12).

Unitary representations of the groups $\mathrm{O}(2, n)$ are of interest in many areas of mathematics and mathematical physics. First, these representations give an example of a quantization of a dynamical system with symmetry. Here we are taking the more traditional point of view, rather than the more general point of view of quantization as defined by Kostant and Souriau, [So.2]. See in this context also the paper by Elhadad [El]. For the case $n=3$, (the original hydrogen atom problem) the dynamical Lie algebra has been extensively studied. The reference [Ba2] contains an extensive bibliography to applications of the dynamical Lie algebras and some of their generalizations to determination of energy spectra of some quantum mechanical systems. Most of the results concerning the dynamical group are of a formal nature (see §2). In fact it isn't clear from such formal considerations in what sense these operators are skew self adjoint. In fact it turns out that there is a Hilbert space defined on suitable function classes on $\mathbb{R}^{n}$ for which appropriate skew self adjoint extensions of these operators are infinitesimal generators of unitary transformations, but such results come out of considerations such as the ones given in this paper. In this effort we apply some recent ideas from the theory of infinite dimensional representations of semisimple Lie groups.

One of the main results of this paper (Theorem 4) is that the dynamical Lie algebra is the Lie algebra of infinitesimal generators of a 
suitable unitary representation of the orthogonal groups $\mathrm{O}(2, n+1)$. We outline the construction as follows.

One considers a representation of the identity component of $\tilde{\mathbb{G}}=$ $\mathrm{O}(2, n+1)$ induced by a not necessarily unitary one dimensional representation of a maximal parabolic subgroup of the group. These one dimensional representations are called pseudo-characters. It turns out that the representation modules for these induced representations may be viewed as sections of a line bundle over a projective $n+1$ space. For "generic" values of the inducing parameter such representations are irreducible. However the representations fail to be irreducible for certain "half-integer" values of this parameter. For each $n$ the representation that is of particular interest in this paper occurs for one of these half integer values. In this case one has an irreducible component having a lowest weight vector. This irreducible component can also be defined as a solution subspace of a wave equation. The dynamical Lie algebra is obtained from a suitable "Poisson" integral transform of the wave equation. The dynamical Lie algebra consists then of differential operators on this light cone corresponding to the infinitesimal action of the group on the solutions of the wave equation.

We outline the paper in more detail.

Section 2 introduces a dynamical Lie algebra for the $n$-dimensional Kepler problem more or less in the spirit of A. O. Barut for the case $n=3$. The proof of the main result is only indicated here, since it is reobtained as a corollary to the considerations later in the paper.

Section 3 is devoted to those properties of the groups $\mathrm{O}(2, n+1)$ and their Lie algebras needed in this paper. In particular the Bruhat decompositions correspnding to the maximal parabolic subgroups are introduced. The relation to the so-called Möbius transformations on projective $n+1$ space with this decomposition is given. The action of the group $\mathrm{O}(2, n+1)$ on projective $n+1$ space which is of major interest in this paper is closely related to the conformal group on the projective compactification of Minkowski space. If the Minkowski metric here is replaced by a Euclidean metric, then the action of the conformal group is precisely what have recently been called the generalized Möbius transformations. See [A1, A2].

In $\S 4$ we define the relevant induced representations, and work out the infinitesimal action explicitly. It is also pointed out here that the Schwartz space on $\mathbb{R}^{n+1}$ is a natural module for the global action of the group (Lemma 6). 
Section 5 is a review of the theory of universal enveloping algebras of the group $G$. It contains the construction of elements which are invariant under a certain subgroup $K$ which is isomorphic to the Lorentz group $\mathrm{SO}(1, n+1)$.

In $\S 6$ differential equations invariant under the subgroups $G$ and $K$ are derived. The principal result in this section determines the pseudocharacter for which the wave equation defines an invariant subspace of the representation space of the induced representation. We also note here that the eigenspace of the $K$-invariant differential equations may all be expressed in terms of solutions of the wave equation for any value of the inducing pseudo-character. This is accomplished by means of a simple transformation. This result gives a simple expression of the class one spherical functions of the generalized Lorentz groups $\mathrm{SO}_{e}(1, n)$ in terms of solutions of the wave equation.

Section 7 contains a discussion of the transformation which maps the representation module defined by solutions of the wave equation onto the representation module consisting of functions defined on the light cone in $\mathbb{R}^{n+1}$. A chief point of this paper is that for the critical pseudo-character, this transform transfers the infinitesimal action of the Lie algebra $\mathbb{G}$ discussed in $\S 3$ onto the dynamical Lie algebra of $\S 2$. The main result here implies Theorem 1 . The transform is given explicitly by Segal [S1] for the case $n=3$.

Finally we use the following notation. The symbols $\mathbb{R}$ and $\mathbb{C}$ denote respectively the field of real and complex numbers. For each positive integer $m \mathbb{R}^{m}$ denotes the space of $m$-tuples viewed as column matrices. For $A$ a matrix of finite size $A^{\mathrm{T}}$ denotes the transpose of this matrix. The notation for the real classical groups used here is from Helgason [He].

2. The dynamical Lie algebra. Let $y=\left(y_{1}, \ldots, y_{n}\right) \in \mathbb{R}^{n}$. Let $($,$) be$ the standard inner product defined by $(x, y)=\sum_{i=1}^{n} x_{i} y_{i}$. As before write $r(y)=\sqrt{(y, y)}$. For $x \Rightarrow p(x)$ a $C^{\infty}$ self mapping of $\mathbb{R}^{n}$, let $p(y) \cdot \nabla$ denote the operator defined on $C^{\infty}$-functions $f$, which in general can be vector valued, by

$$
p(x) \cdot \nabla f(x)=\left.\frac{d}{d t} f(x+t p(x))\right|_{t=0} .
$$

Considered as operators on $C^{\infty}\left(\mathbb{R}^{n}\right)$ they are just the globally defined vector fields on $\mathbb{R}^{n}$. Also for $i=1, \ldots, n$ let $\left\{e_{1}, \ldots, e_{n}\right\}$ be the natural basis of $\mathbb{R}^{n}$. Consider vectors as constant vector valued functions. Then as a special case one has $e_{i} \cdot \partial=\partial_{i}=\partial / \partial y_{i}$. For any $C^{\infty}$-map 
$p$ from $\mathbb{R}^{n}$ to itself denote by $p^{\prime}(x)$ its derivative at $x \in \mathbb{R}^{n}$. Thus for each $x, p^{\prime}(x)$ is a linear endomorphism of $\mathbb{R}^{n}$. One notes for future reference, that one has the following commutation relations for the operators defined above:

$$
\begin{aligned}
{[p(x) \cdot \nabla, q(x) \cdot \nabla] } & =\left(q^{\prime}(x) p(x)\right) \cdot \nabla-\left(p^{\prime}(x) q(x)\right) \cdot \nabla \\
& =(q(x) \cdot \nabla p(x)) \cdot \nabla-(p(x) \cdot \nabla q(x)) \cdot \nabla .
\end{aligned}
$$

We consider the operation of multiplication by a $C^{\infty}$-function as a differential operator of order 0 . Consider the associative algebra of differential oeprators on $\mathbb{R}^{n}$ generated by these multiplications and the vector fields. The differential operators also form a Lie algebra under commutation: $[A, B]=A B-B A$. We shall define a finite dimensional subspace $\mathbf{G}$ of this algebra which will turn out to be a Lie subalgebra.

First define for $i, j=1, \ldots, n$

$$
L_{i j}=y_{i} \partial_{j}-y_{j} \partial_{i}
$$

It is clear that $L_{i j}=-L_{j i}$ and $L_{i i}=0$. We also define the Laplacian:

$$
\Delta=\partial_{1}^{2}+\cdots+\partial_{n}^{2},
$$

and the so-called Euler operator in the above notation is $E=y \cdot \partial ; y$ here is considered as the identity map. In more traditional notation,

$$
E=\sum_{i=1}^{n} y_{i} \partial_{i}
$$

Finally the "symmetrized" Euler operator is defined as

$$
F=\frac{1}{2} \sum_{i=1}^{n}\left(y_{i} \partial_{i}+\partial_{i} y_{i}\right) \text {. }
$$

It is also clear that $F=E+\frac{1}{2} n \mathbf{1}$. Finally, one defines the following real linear subspaces of the algebra of differential operators:

$$
\begin{aligned}
& \mathbf{W}_{+}=\operatorname{span}_{\mathbf{R}}\left\{\partial_{i}\left(F-\frac{1}{2} \mathbf{1}\right)-\frac{1}{2} \Delta y_{i}, r \Delta: i=1, \ldots, n\right\}, \\
& \mathbf{W}_{0}=\mathbf{M}+\mathbf{P}_{1}+\mathbb{R}\left(F-\frac{1}{2} \mathbf{1}\right),
\end{aligned}
$$

where

$$
\mathbf{M}=\operatorname{span}_{\mathbf{R}}\left\{L_{i j}: 1 \leq i<j \leq n\right\},
$$

and

$$
\begin{aligned}
\mathbf{P}_{1} & =\operatorname{span}_{\mathbb{R}}\left\{r \partial_{i}: i=1, \ldots, n\right\}, \\
\mathbf{W}_{-} & =\operatorname{span}_{\mathbb{R}}\left\{y_{j}, r: j=1, \ldots, n\right\} .
\end{aligned}
$$


Define $\mathbf{G}$ as the space spanned by $\mathbf{W}_{+}, \mathbf{W}_{0}$, and $\mathbf{W}_{-}$:

$$
\mathbf{G}=\mathbf{W}_{+}+\mathbf{W}_{0}+\mathbf{W}_{-} .
$$

LEMMA 1. One has the following commutation rules between the sets of differential operators defined above and the individual operators:

(1) The vector space direct sum $\mathbf{G}=\mathbf{W}_{+}+\mathbf{W}_{0}+\mathbf{W}_{-}$is direct; in fact, it is the eigenspace decomposition of $\mathbb{G}$ under the linear mapping $X \Rightarrow$ $\left[-\left(F-\frac{1}{2} \mathbf{1}\right), X\right]$, with eigenvalues $+1,0-1$. That is, $\left[-\left(F-\frac{1}{2} \mathbf{1}\right), X\right]=$ $X, 0,-X$ depending on whether $X$ is in $\mathbf{W}_{+}, \mathbf{W}_{0}$, or $\mathbf{W}_{-}$respectively.

$$
\begin{aligned}
& {\left[\mathbf{W}_{+}, \mathbf{W}_{+}\right]=\{0\}=\left[\mathbf{W}_{-}, \mathbf{W}_{-}\right],} \\
& {\left[\mathbf{W}_{0}, \mathbf{W}_{0}\right]=\mathbf{W}_{0}, \quad\left[\mathbf{W}_{0}, \mathbf{W}_{+}\right]=\mathbf{W}_{+}, \quad\left[\mathbf{W}_{0}, \mathbf{W}_{-}\right]=\mathbf{W}_{-},} \\
& {\left[\mathbf{W}_{+}, \mathbf{W}_{-}\right]=\mathbf{W}_{0},}
\end{aligned}
$$

In particular, the subspace $\mathbf{G}$ is a Lie subalgebra of the Lie algebra of differential operators under the Lie bracket [, ] multiplication.

(3) The linear subspace $\mathbf{M}+\mathbf{P}_{1}$ is a Lie subalgebra.

(4) The sum defining the algebra in (3) is direct, and gives a Cartan decomposition:

$$
\left[\mathbf{P}_{1}, \mathbf{P}_{1}\right]=\mathbf{M}, \quad\left[\mathbf{P}_{1}, \mathbf{M}\right]=\mathbf{P}_{1}, \quad[\mathbf{M}, \mathbf{M}]=\mathbf{M} .
$$

In particular, $\mathbf{M}$ is a subalgebra.

Proof. All assertions concerning the structure of $\mathbf{G}$ as a Lie algebra can be checked from (1) and from the following observation: If $A, B, C$ are elements of any associative algebra, then $[A, B C]=$ $[A, B] C+B[A, C]$. Thus $X \Rightarrow[A, X]$ is a derivation. It follows from this remark that if $p(y)$ is a homogeneous polynomial of degree $r$, and $q(\partial)$ is a homogeneous polynomial of degree $s$ in the operators $\partial_{i}$, then

$$
\left[\left(F-\frac{1}{2} \mathbf{1}\right), p(y) q(\partial)\right]=(r-s) p(y) q(\partial) .
$$

This results immediately in (1). The derivation property referred to above results also in the second and third lines of (2). One also has $\left[\partial_{i}, y_{j}\right]=\delta_{i j},\left[\partial_{i}, r\right]=y_{i} / r$, and $\left[\Delta, y_{i}\right]=2 \partial_{i}$, and $[\Delta, r]=$ $(1 / r)(2 F-1)$. With these observations one can also verify $\left[\mathbf{W}_{+}, \mathbf{W}_{+}\right]=$ $\{0\}$. Since we will have other means of seeing this, the slightly messy details are left to the reader. The observation that $\left[\mathbf{W}_{-}, \mathbf{W}_{-}\right]=\{0\}$ is 
obvious. The above considerations lead easily to

$$
\left[r \partial_{i}, r \partial_{j}\right]=y_{i} \partial_{j}-y_{j} \partial_{j}=L_{i j}
$$

This implies the first relation in (4). The remaining assertions can be verified in a similar spirit. However, the following short cut makes them obvious. For $T$ a rotation, let $\tilde{\mathbf{T}}$ denote the operator on $C^{\infty}\left(\mathbb{R}^{n}\right)$ defined by $\tilde{\mathbf{T}} f(y)=f\left(T^{-1} y\right)$ for $y \Rightarrow T y$ the usual linear action. One checks by standard arguments that for $1 \leq i<j \leq n, L_{i j}$ is the vector field associated with the rotations in the 2-plane spanned by $e_{i}$ and $e_{j}$. (See proof of Theorem 1 below.) Since it is known that these infinitesimal rotations span the Lie algebra so( $n), \mathbf{M}$ must be isomorphic to the Lie algebra so $(n)$. One considers the action $D \Rightarrow \tilde{\mathbf{T}}^{-1} D \tilde{\mathbf{T}}$ of the rotations on the differential operators. It is then easy to check that the operators $\left(F-\frac{1}{2} \mathbf{1}\right), \Delta$, and $r$ are invariant under this action. It follows also that the operators $\partial_{i}\left(F-\frac{1}{2} \mathbf{1}\right)-\frac{1}{2} \Delta y_{i}, r \partial_{i}$, and $y_{i}$ transform like the vectors in $\mathbb{R}^{n}$ under the rotations. By differentiating along the one parameter subgroups of the rotations one gets the remaining assertions of the lemma.

One can now establish an isomorphism from the algebra $\mathbf{G}$ onto the Lie algebra so $(2, n+1)$. For this purpose let $B(\cdot, \cdot)$ be a non-degenerate bilinear form on $\mathbb{R}^{n+3}$ defined by

$$
\mathbf{B}(x, y)=-x_{-1} y_{-1}-x_{0} y_{0}+x_{1} y_{1}+\cdots+x_{n} y_{n}+x_{n+1} y_{n+1}
$$

for $x, y \in \mathbb{R}^{n+3}$. The Lie algebra $\operatorname{so}(2, n+1)$ is defined as the set of linear endomorphisms $T$ of $\mathbb{R}^{n+3}$ that satisfy $\mathbf{B}(T x, y)=-\mathbf{B}(x, T y)$. Let $E_{i j}=\mathbf{e}_{1} \mathbf{e}_{j}^{\mathrm{T}}$ be the matrix units for $-1 \leq i, j \leq n+1$. From the above definition one checks easily the standard fact that $\operatorname{so}(2, n+1)$ has as a linear basis the endomorphisms:

$$
\begin{aligned}
\left\{E_{i j}-E_{j i}, E_{0-1}-E_{-10}, E_{0 k}+E_{k 0}, E_{-1 k}+E_{k,-1}:\right. & \\
1 & \leq i<j \leq n+1,1 \leq k \leq n+1\} .
\end{aligned}
$$

Let $X_{i j}=E_{i j}-E_{j i}$, for $1 \leq i<j \leq n$. These are the elements of the above basis that are to correspond to the infinitesimal rotations in the "original" $n$-space. Let $X=-E_{-10}+E_{0-1}$ and for $1 \leq k \leq n$, $X_{k}=E_{k, n+1}-E_{n+1, k}$. These then are the additional infinitesimal rotations of the above basis. Let for $1 \leq k \leq n, Y_{k}=E_{-1, k}+E_{k,-1}$, $Z_{k}=E_{0, k}+E_{k, 0}, H=Y_{-1, n+1}:=E_{n+1,-1}+E_{-1, n+1}$ and $Y=Y_{0, n+1}:=$ $E_{0, n+1}+E_{n+1,0}$. These are the infinitesimal hyperbolic rotations of the above basis. 
The relevant facts can be summarized in the following:

THEOREM 1. There is an isomorphism $\Psi$ of $\mathbf{G}$ onto so $(2, n+1)$ which maps the basis elements given above according to the following table:

$$
\begin{array}{lc}
H & -\left(F-\frac{1}{2} \mathbf{1}\right) \\
Y-X & -i r \Delta \\
Y+X & -i r \\
X_{i j} & L_{i j} \\
Z_{k} & r \partial_{k} \\
Y_{k}-X_{k} & -2 i\left(\partial_{k}\left(F-\frac{1}{2} \mathbf{1}\right)-\frac{1}{2} \Delta y_{k}\right) \\
Y_{k}+X_{k} & -i y_{k}
\end{array}
$$

where $i=\sqrt{-1}$.

Proof. The above table defines by linearity a linear isomorphism from $\mathbb{G}$ onto $\operatorname{so}(2, n+1)$. The main point then is to check that the commutation rules are preserved under the map $\Psi$. Again, since we shall present global methods for verifying that $\Psi$ is a Lie algebra isomorphism, only the main points are sketched. One essential short cut has already been pointed out in the proof of Lemma 1, namely to consider the action of the rotations. First one observes that if $f \in C^{\infty}\left(\mathbb{R}^{n}\right)$, then

$$
L_{i j} f=d \exp \left(-t X_{i j}\right) f /\left.d t\right|_{t=0} .
$$

One notes next that under the representation of the rotations given by $D \Rightarrow \tilde{\mathbf{T}}^{-1} D \tilde{\mathbf{T}}$, the subsets $\left\{r \partial_{j}\right\},\left\{-x_{k}\right\}$, and $\left(\partial_{k}\left(F-\frac{1}{2} \mathbf{1}\right)-\frac{1}{2} \Delta y_{k}\right)$ transform as the vectors in $\mathbb{R}^{n}$ under the usual action of the rotations, while the elements $r \Delta, r$, and $\left(F-\frac{1}{2} \mathbf{1}\right)$ are invariant. From this observation it is a standard argument to show that the commutation relations of the $X_{i j}$ with the remaining elements of the above basis of $\operatorname{so}(2, n+1)$ are preserved by the mapping $\Psi$.

The commutation relations with the element $-\left(F-\frac{1}{2} \mathbf{1}\right)$ were pointed out in Lemma 1. These correspond to the following relations for the matrices: $[H, Y \pm X]=\mp(Y \pm X),\left[H, Y_{k} \pm X_{k}\right]=\mp\left(Y_{k} \pm X_{k}\right)$, $\left[H, Z_{k}\right]=0$, and $\left[H, L_{i j}\right]=0$. 
The techniques mentioned in the proof of the above lemma lead to the formulas:

$$
\begin{gathered}
{\left[\partial_{k}\left(F-\frac{1}{2} \mathbf{1}\right)-\frac{1}{2} \Delta y_{k}, r \partial_{j}\right]=\frac{1}{2} \delta_{j k} r \Delta,} \\
{\left[r \Delta, r \partial_{i}\right]=2\left(\partial_{i}\left(F-\frac{1}{2} \mathbf{1}\right)-\frac{1}{2} \Delta y_{i}\right),} \\
{\left[r, r \partial_{i}\right]=-y_{i},} \\
{\left[y_{i}, r \partial_{k}\right]=-\delta_{i k} r,} \\
{[r \Delta, r]=2\left(F-\frac{1}{2} \mathbf{1}\right),} \\
{\left[r \Delta, y_{k}\right]=2 r \partial_{k},} \\
{\left[\partial_{k}\left(F-\frac{1}{2} \mathbf{1}\right)-\frac{1}{2} \Delta y_{k}, y_{k}\right]=\left(F-\frac{1}{2} \mathbf{1}\right),} \\
{\left[\partial_{k}\left(F-\frac{1}{2} \mathbf{1}\right)-\frac{1}{2} \Delta y_{k}, y_{j}\right]=L_{j k}, \quad j \neq k,} \\
{\left[\partial_{k}\left(F-\frac{1}{2} \mathbf{1}\right)-\frac{1}{2} \Delta y_{k}, r\right]=r \partial_{k}+\left[\partial_{k}, r\right]-y_{k} / r=r \partial_{k} .}
\end{gathered}
$$

The commutators not indicated are 0 . Again the details are left to the reader. These formulae make explicit (2) of Lemma 1. One can then check easily that (2.2) together with the above list of commutation rules correspond, to the following matrix commutation rules: $(2.2) \Leftrightarrow$ $\left[Z_{i}, Z_{j}\right]=X_{i j},(2.3) \Leftrightarrow\left[Y_{k}-X_{k}, Z_{j}\right]=-\delta_{k j}(Y-X),(2.4) \Leftrightarrow[Y-X$, $\left.Z_{k}\right]=Y_{k}-X_{k},(2.5) \Leftrightarrow\left[Y+X, Z_{k}\right]=-\left(Y_{k}+X_{k}\right),(2.6) \Leftrightarrow\left[Y_{k}+X_{k}\right.$, $\left.Z_{j}\right]=-\delta_{k j}(Y+X),(2.7) \Leftrightarrow[Y-X, Y+X]=2 H,(2.8) \Leftrightarrow[Y-X$, $\left.X_{k}+Y_{k}\right]=-2 Z_{k},(2.9 \mathrm{a}) \Leftrightarrow\left[Y_{k}-X_{k}, Y_{j}+X_{j}\right]=2 X_{j k},(2.9 \mathrm{~b}) \Leftrightarrow$ $\left[Y_{k}-X_{k}, Y_{k}+X_{k}\right]=2 H,(2.10) \Leftrightarrow\left[Y_{k}-X_{k}, Y+X\right]=-2 Z_{k}$. This establishes the theorem.

One remarks that the table in the theorem implies in particular that the elements $X_{k}$ commute with the element $X$. The latter represents the Hamiltonian operator for the case of the negative energy levels, while the elements $Y_{k}$ commute with the element $Y$, which is the Hamiltonian operator for the positive energy levels.

3. The orthogonal groups $\mathrm{O}(2, n+1)$ and their Lie algebras so $(2, n+1)$; description of the conformal groups on Minkowski spaces and Möbius transformations. Let $J$ be the $(n+3) \times(n+3)$ matrix given by $\operatorname{diag}[-1,1,1, \ldots, 1]$. Then it is clear that $J^{2}=1$, so that the map $X \Rightarrow \Theta(X)=J X J$ is an involution on the general linear group $\mathrm{Gl}(n+3, \mathbb{R})$. Let $B$ be the matrix $B=\operatorname{diag}[-1,-1,1, \ldots, 1]$, and let the bilinear form $\mathbf{B}$ be defined by $\mathbf{B}(x, y)=x^{\mathrm{T}} B y, x, y \in \mathbb{R}^{n+3}$. (Recall that for any matrix $A, A^{\mathrm{T}}$ denotes its transpose.) Then the group $\mathrm{O}(2, n+1)$ is by definition the invariance group of the form $\mathbf{B}$. In 
terms of the matrix $B$, this definition is equivalent to the definition:

$$
\begin{aligned}
\mathrm{O}(2, n+1) & =\left\{g \in \mathrm{Gl}(n+3, \mathbb{R}): g^{\mathbf{T}} B g=B\right\} \\
& =\left\{g \in \mathrm{Gl}(n+3, \mathbb{R}): g^{-1}=B g^{\mathbf{T}} B\right\} .
\end{aligned}
$$

The second condition follows easily from the first by the fact that $B^{2}=1$.

Let $G=\mathrm{SO}_{e}(2, n+1)$, the identity component of $\mathrm{O}(2, n+1)$. In any topological group the identity component is a normal subgroup of the group. In the present case it is known that $\mathrm{O}(2, n+1)$ has four components; equivalently, the quotient group $\mathrm{O}(2, n+1) / \mathrm{SO}_{e}(2, n+1)$ is of order four. More precisely, this quotient is the direct product of two cyclic groups of order two. (See Lemma 4.)

It is clear that $J B=B J$. One deduces from this fact that the involution $\Theta$ stabilizes the group $\mathrm{O}(2, n+1)$. Thus $\Theta$ also defines an involution on the latter group which will also be denoted by $\Theta$. By differentiating along the one parameter subgroups $t \nrightarrow \exp (t X)$, one sees that $\operatorname{so}(2, n+1)$ as defined in the previous section is the Lie algebra of $\mathrm{O}(2, n+1)$. Let $\mathbb{G}$ denote this Lie algebra. Its definition may be recapitulated in terms of $B$ as follows:

$$
\begin{aligned}
\mathbb{G} & =\left\{X \in \mathbb{R}_{n+3}^{n+3}: \mathbf{B}(X y, y)=-\mathbf{B}(y, X y), \forall y \in \mathbb{R}^{n+3}\right\} \\
& =\left\{X \in \mathbb{R}_{n+3}^{n+3}: X^{\mathbf{T}} \mathbf{B}=-\mathbf{B} X\right\} .
\end{aligned}
$$

The map $\Theta$ acts as an involution on the Lie algebra $\mathbb{G}$. Again denoting this involution by the same letter as before, one has $\Theta(\exp (X))=$ $\exp (\Theta(X))$. This point is easy to check from the exponential series and the fact that $J^{2}=1$. On the Lie algebra level let $\mathbb{L}$ and $\mathbb{K}$ denote the -1 and +1 eigenspaces respectively of the linear map $X \Rightarrow \Theta(X)$. Since this map is involutive, it is semisimple and these are the only eigenspaces. Hence one has the direct sum: $\mathbb{G}=\mathbb{L} \oplus \mathbb{K}$. Let $\mathbb{W}_{0}, \mathbb{W}_{ \pm}, \mathbb{M}$ and $\mathbb{P}$ denote the subspaces and subalgebras corresponding respectively, under the isomorphism of Lemma 1, to the spaces of operators denoted by the corresponding bold face letters. In terms of the matrices defined above one has explicitly:

$$
\begin{aligned}
\mathbb{M} & =\operatorname{span}\left\{X_{i j}: 1 \leq i<j \leq n\right\}, \\
\mathbb{K} & =\operatorname{span}\left\{Z_{k}, X_{k}, Y: 1 \leq k \leq n\right\} \oplus \mathbb{M}, \\
\mathbb{L} & =\operatorname{span}\left\{Y_{k}, H, X: 1 \leq k \leq n\right\}, \\
\mathbb{P}_{1} & =\operatorname{span}\left\{Z_{k}: 1 \leq k \leq n\right\}, \\
\mathbb{W}_{0} & =\mathbb{R} H \oplus \mathbb{M} \oplus \mathbb{P}_{1}, \\
\mathbb{W}_{ \pm} & =\operatorname{span}\left\{Y \mp X, Y_{k} \mp X_{k}: 1 \leq k \leq n\right\} .
\end{aligned}
$$


Also set

$$
\mathbb{P}_{2}=\operatorname{span}\left\{Y_{k}: 1 \leq k \leq n\right\}, \quad \mathbb{P}_{3}=\operatorname{span}\left\{X_{k}: 1 \leq k \leq n\right\} .
$$

Then define $M_{1}, M_{2}$ and $M_{3}$ by

$$
\mathbf{M}_{1}:=\mathbf{M} \oplus \mathbb{P}_{1}, \quad \mathbf{M}_{2}:=\mathbb{P}_{2} \oplus \mathbf{M}, \quad \mathbf{M}_{3}:=\mathbb{P}_{3} \oplus \mathbf{M} .
$$

Clearly then the involution $\Theta$ fixes the subalgebra $W_{0}$ and interchanges the spaces $\mathbb{W}_{+}$and $\boldsymbol{W}_{-}$. One notices that because the $\mathbb{P}_{j}$ for $j=1,2,3$, transform like $\mathbb{R}^{n}$ under the adjoint representation of the rotations, one has $\left[\mathbb{P}_{j}, \mathbf{M}\right]=\mathbb{P}_{j}, j=1,2,3$. Thus the $\mathbf{M}_{j}$ are subalgebras of $\mathbb{G}$. It follows easily from the definitions that $M_{1}$ and $M_{2}$ are isomorphic to so $(1, n)$, while $\mathrm{M}_{3}$ is isomorphic to so $(n+1)$.

Set $\mathbb{G}_{1}=\operatorname{span}\{X, Y, Z\}$.

Proposition 1. The space $\mathbb{G}_{1}$ is a subalgebra, isomorphic to so $(1,2)$. $\mathrm{M}_{2}$ and $\mathrm{M}_{3}$ are isomorphic to so $(1, n)$ and so $(n+1)$ respectively, and $\mathrm{M}_{1}$ is isomorphic to so $(1, n)$. The direct sums defining these algebras are Cartan decomposition. This means that $\left[\mathbb{P}_{i}, \mathbb{P}_{i}\right]=\mathbf{M}$, and $\left[\mathrm{M}, \mathbb{P}_{i}\right]=\mathbb{P}_{i}$, $i=1,2,3$. Moreover, $\mathbf{W}_{0}=\mathbb{R} H \oplus \mathbf{M}_{1}$ is the centralizer of $H$ in $\mathbb{G}$, $\mathbb{R} Y \oplus \mathbf{M}_{2}$ is the centralizer of $Y$ in $\mathbb{G}$, and $\mathbb{R} X \oplus \mathrm{M}_{3}$ is the centralizer of $X$ in $\mathbb{G}$. The latter centralizer is a maximal compact subalgebra of $\mathbb{G}$. The algebra $\mathbb{K}$ is isomorphic to so $(1, n+1)$ and is a maximal semisimple subalgebra of $\mathbb{G}$. The direct sum $\mathbb{G}=\mathbb{L} \oplus \mathbb{K}$ is a Cartan decomposition, exhibiting $(\mathbb{G}, \mathbb{K})$ as a non-Riemannian symmetric pair whose Cartan involution is $\Theta$. The major inclusions and isomorphisms are summarized in the diagram below:

$$
\begin{aligned}
\mathbb{R}^{n+1} \cong & \mathbb{W}_{-} \\
& \cap \\
& \mathbb{G} \supset \mathbb{K} \cong \operatorname{so}(1, n+1) \supset M_{1} \cong \operatorname{so}(1, n) \supset \mathbb{M} \\
& \mathbb{G} \supset \mathbb{W}_{0}=M_{1} \oplus \mathbb{R} H \supset \mathbb{M} \cong \operatorname{so}(n) \\
& \mathbb{G} \supset \mathbb{R} Y \oplus M_{2} \supset \mathbb{M} \\
& \cup \mathbb{K} \supset M_{3} \cong \operatorname{so}(n+1) \supset \mathbb{M} \\
\mathbb{R}^{n+1} \cong & \mathbb{W}_{+}
\end{aligned}
$$

Proof. These results follow in a straightforward manner from the commutation rules for the spanning elements of the Lie algebra $G$, pointed out in the proof of Theorem 1 .

The following remark is also useful in our analysis. 
Proposition 2. The centralizers of the elements $H, Y$, and $X$ are the algebras $\mathbb{R} H \oplus \mathrm{M}_{1}, \mathbb{R} Y \oplus \mathrm{M}_{2}$, and $\mathbb{R} X \oplus \mathrm{M}_{3}$ respectively.

Proof. To check that the centralizer of $H$ is as asserted, one notes that the elements $Z_{k}$ are the only spanning elements of $\mathbb{G}$, other than $H$ itself, that commute $H$. The assertion follows from the definition of the subalgebra $M_{1}$ and by linearity. The other assertions follow in a similar manner.

In order to analyze the component structure of certain subgroups of $G$ we need the following lemmas:

LEMMA 2 (Polar decomposition). Let $\mathrm{O}(p, q)$ be the orthogonal group of a real quadratic form with signature $p+$ and $q-$. Assume $0<p \leq q$. Then one has the polar decomposition $\mathrm{O}(p, q)=\tilde{Q} S$, where $S$ consists of the positive symmetric elements in $\mathrm{O}(p, q)$, and $\tilde{Q}$ consists of the orthogonal elements in $\mathrm{O}(p, q)$. One has $\tilde{Q} \cap S=\{1\}$.

Proof. This follows from standard arguments in linear algebra. (See for example [Th].)

LEMMA 3. Let $Q$ be the identity component of $\tilde{Q}$. Let $J_{1}, J_{2}$, and $J_{3}$ be the matrices defined by $J_{1}=\operatorname{diag}\left[-1,1_{p+q-1}\right], J_{2}=\operatorname{diag}\left[1_{p+q-1},-1\right]$, and $J_{3}=J_{1} J_{2} . Q$ is a normal subgroup of $\tilde{Q}$, and the quotient group $\tilde{Q} / Q$ consists of the four cosets $Q, J_{1} Q, J_{2} Q$, and $J_{3} Q$. Thus $\tilde{Q} / Q$ is isomorphic to the direct product of two cyclic groups of order 2 .

Proof. We realize the group $\mathrm{O}(p, q)$ as the set of $(p+q) \times(p+q)$ matrices $A$ such that $A^{\mathrm{T}} C A=C$, where $C$ is a diagonal matrix with diagonal entries: $C_{i i}=-1$, if $1 \leq i \leq p$, and $C_{i i}=1$, if $p+1 \leq$ $i \leq p+q$. If in addition to belonging to $\mathrm{O}(p, q), A$ is orthogonal, then $A^{\mathbf{T}} A=1_{p+q}=A A^{\mathrm{T}}$. Thus by pre-multiplication of the defining condition by $A$, one sees that $A C=C A$. Using partitioned matrices one checks that $\tilde{Q}$ is given by the matrices of the form: $\operatorname{diag}[\alpha, \delta]$, with $\alpha$ and $\delta p \times p$ and $q \times q$ orthogonal matrices respectively. Thus $\tilde{Q}$ is isomorphic to $\mathrm{O}(p) \times \mathrm{O}(q)$. Now it is known that for any positive integer $m$ orthogonal group $\mathrm{O}(m)$ has two connected components. The identity component consists of those elements with determinant equal to one. The component that does not contain the identity consists of the matrices with determinant -1 . As a coset representative for this latter component one may take any element in $\mathrm{O}(m)$ of determinant -1 . The lemma follows then easily from these remarks. 
LEMMA 4. Let $\mathrm{SO}_{e}(p, q)$ denote the identity component of $\mathrm{O}(p, q)$. This identity component is a normal subgroup of $\mathrm{O}(p, q)$, and the quotient group $\mathrm{O}(p, q) / \mathrm{SO}_{e}(p, q)$ consists of the four cosets $\mathrm{SO}_{e}(p, q)$, $J_{1} \mathrm{SO}_{e}(p, q), J_{2} \mathrm{SO}_{e}(p, q)$, and $J_{3} \mathrm{SO}_{e}(p, q)$.

Proof. It is known that every element of $S$ is the exponential of a symmetric element in $\mathrm{O}(p, q)$. Thus $S$ is homeomorphic to the Euclidean space consisting of the symmetric elements of the Lie algebra $\mathbb{G}$. Thus the space consisting of the symmetric elements of the Lie algebra $\mathbb{G}$. Thus the lemma follows from the polar decomposition (Lemma 2).

Let the analytic subgroups corresponding to the above Lie subalgebras be denoted by the corresponding plain capital letter. Let $\tilde{K}$ denote the centralizer of the matrix $J$. From the definition of the involution $\Theta$, one has equivalently that $\tilde{K}$ is the subgroup of $G$ which is elementwise fixed under this involution. Since the subalgebra $\mathbb{K}$ is the fixed point subalgebra under $\Theta$, it follows that $K \subset \tilde{K}$. Moreover, since all of the matrices in $\mathbb{K}$ have zeros in the first row and column, one has explicitly:

$$
K=\left\{\operatorname{diag}[1, k]: k \in \mathrm{SO}_{e}(1, n+1)\right\} .
$$

Incidentally, this also shows that $\mathbb{K}$ is isomorphic to $\operatorname{so}(1, n+1)$ as pointed out in Proposition 1. On the other hand, one checks that

$$
\tilde{K}=\{\operatorname{diag}[ \pm 1, k]: k \in \mathrm{O}(1, n+1)\} \cap \mathrm{SO}_{e}(2, n+1) .
$$

Note that $B$ is in the identity component of $G$. In fact $B$ is in the one parameter subgroup

$$
\theta \nrightarrow \operatorname{diag}\left(\left(\begin{array}{cc}
\cos (\theta) & -\sin (\theta) \\
\sin (\theta) & \cos (\theta)
\end{array}\right), 1, \ldots, 1\right)
$$

with $\theta=\pi$. However the element $B_{1}$ defined by

$$
B_{1}=\operatorname{diag}\left[-1,1_{n+1},-1\right]
$$

is not in $G$. By specializing Lemma 4 to the case $p=2$, one obtains that $\tilde{K}$ has the coset decomposition:

$$
\tilde{K}=K \cup B K
$$

In paticular, $\tilde{K}$ has two components. 
Let $\tilde{M}_{1}$ denote the centralizer of the matrix $H$ in $\tilde{K}$. One checks by the definition that the centralizer of $H$ must have the following block matrix form:

$$
\left(\begin{array}{ccccc}
a & 0 & \ldots & 0 & b \\
0 & & & & 0 \\
\vdots & & m & & \vdots \\
0 & & & & 0 \\
b & 0 & \ldots & 0 & a
\end{array}\right)
$$

with $a, b \in \mathbb{R}$, and $m$ an $n+1$ by $n+1$ matrix. However in order for such an element to be in $\tilde{K}$, one must have from the above considerations, $a= \pm 1, b=0$. Now since $\mathrm{M}_{1}$ is the centralizer of $H$ in $\mathbb{K}$, as pointed out in Proposition 1, one has that $M_{1} \subset \tilde{M}_{1}$. Since the matrices in $\mathbf{M}_{1}$ have no non-zero elements in rows and columns numbered -1 , and $n+1$, it follows that the elements of $\tilde{M}_{1}$ have the above form with $a=1, b=0$. Identifying $M_{1}$ with $\mathrm{SO}_{e}(1, n)$ for the moment, one finds from Lemma 3 that a complete set of representatives of the component group consists of the matrices $1_{n+1}, \operatorname{diag}\left[-1,1_{n}\right]$, diag $\left[1_{n},-1\right]$, and diag $\left[-1,1_{n-1},-1\right]$. However, only the identity matrix and the last matrix can be fit into (3.1) to produce a matrix in $G$. Hence writing $J_{1}=\operatorname{diag}\left[-1,-1,1_{n-1},-1,-1\right]$, one has established the following:

Proposition 3. For the centralizer $\tilde{M}_{1}$ of $H$ one has the coset decomposition: $\tilde{M}_{1}=M_{1} \cup J_{1} M_{1}$. In particular, $\tilde{M}_{1}$ consists of two components.

Proposition 4. Let $A=\{\exp (t H): t \in \mathbb{R}\}$, and $P_{ \pm}=W_{ \pm} A \tilde{M}_{1}$. Then $P_{ \pm}$are subgroups of $G$. They are parabolic in the sense that they are their own normalizers in $G$, and are maximal with respect to this property. $A \tilde{M}_{1}$ is a normal subgroup of $P_{ \pm}$, and $A$ is the center of $\tilde{M}_{1}$.

For $a \in A, m \in M$, and $n \in W_{+}$, one has $a m n=m n^{a} a$, where $n^{a}=a_{n a}^{-1} \in W_{+}$.

Let $C$ be any matrix in $G$ with the properties $C^{2}=1$, and that $C H C=-H$.

Proposition 5 (Bruhat decompositions). The group $G$ can be expressed in terms of unions of products:

$$
G=W_{-} A \tilde{M}_{1} W_{+} \cup C A \tilde{M}_{1} W_{+} .
$$

Proof. This is proved elsewhere. See [Th]. 
For each $x \in \mathbb{R}^{n+1}$ one defines the elements $V(x)$ by

$$
V(x)=x_{0}(Y+X)+x_{1}\left(Y_{1}+X_{1}\right)+\cdots+x_{n}\left(Y_{n}+X_{n}\right),
$$

and $N(x)=\Theta(V(x))$. Hence, the abelian subalgebras $\mathbb{W}_{-}$and $\mathbb{W}_{+}$ may be expressed as

$$
W_{+}=\left\{N(x): x \in \mathbb{R}^{n+1}\right\}, \quad \text { and } \quad W_{-}=\left\{V(x): x \in \mathbb{R}^{n+1}\right\} .
$$

Explicitly,

$$
N(x)=x_{0}(Y-X)-x_{1}\left(Y_{1}-X_{1}\right)-\cdots-x_{n}\left(Y_{n}-X_{n}\right) .
$$

The matrices $V(x)$ and $N(x)$ may also be expressed as follows:

$$
\begin{aligned}
V(x)= & {\left[\begin{array}{cccccc}
0 & -x_{0} & x_{1} & \ldots & x_{n} & 0 \\
x_{0} & & & & & x_{0} \\
x_{1} & & & & & x_{1} \\
\vdots & & & \mathbb{O}_{n+1}^{n+1} & & \vdots \\
x_{n} & & & & & x_{n} \\
0 & x_{0} & -x_{1} & \ldots & -x_{n} & 0
\end{array}\right] } \\
= & {\left[\begin{array}{ccc}
0 & x^{\mathbf{T}} B_{1} & 0 \\
x & \mathbb{O}_{n+1}^{n+1} & x \\
0 & -x^{\mathbf{T}} B_{1} & 0
\end{array}\right], } \\
& N(x)=\left[\begin{array}{ccc}
0 & -x^{\mathbf{T}} B_{1} & 0 \\
-x & \mathbb{O}_{n+1}^{n+1} & x \\
0 & -x^{\mathbf{T}} B_{1} & 0
\end{array}\right] .
\end{aligned}
$$

where $B_{1}$ is the $n+1$ by $n+1$ matrix given by $B_{1}=\operatorname{diag}[-1,1, \ldots, 1]$. Define the real symmetric form defined on $\mathbb{R}^{n+1}$ by

$$
\langle x, y\rangle=-x_{0} y_{0}+x_{1} y_{1}+\cdots+x_{n} y_{n} .
$$

Notice that the matrix $B_{1}$ is the matrix of the form $\langle$,$\rangle ; that is$ $\langle x, y\rangle=x^{\mathrm{T}} B_{1} y$.

Next, for $t \in \mathbb{R}$, and for $x \in \mathbb{R}^{n+1}$ define elements $a(t) \in A, v(x) \in$ $W_{\text {- by }} a(t)=\exp (t H)$, and

$$
v(x)=\exp (V(x))=\exp \left(x_{0}(Y+X)+\sum_{j=1}^{n} x_{j}\left(Y_{j}+X_{j}\right)\right) .
$$

Define the $n(x) \in W_{+}$by $n(x)=\Theta(v(x))$. 
By using the observation that the matrices $N(x)$ and $V(x)$ are nilpotent of order 2, and from the Maclaurin series for cosh and sinh, one computes explicitly:

$$
\begin{gathered}
v(x) a(t)=\left[\begin{array}{ccc}
\cosh (t)+\frac{1}{2} e^{t}\langle x, x\rangle & x^{\mathbf{T}} B_{1} & \sinh (t)+\frac{1}{2} e^{t}\langle x, x\rangle \\
e^{t} x & 1_{n+1} & e^{t} x \\
\sinh (t)-\frac{1}{2} e^{t}\langle x, x\rangle & -x^{\mathbf{T}} B_{1} & \cosh (t)-\frac{1}{2} e^{t}\langle x, x\rangle
\end{array}\right] \\
n(x)=\left[\begin{array}{ccc}
1+\frac{1}{2}\langle x, x\rangle & -x^{\mathbf{T}} B_{1} & -\frac{1}{2}\langle x, x\rangle \\
-x & 1_{n+1} & x \\
\frac{1}{2}\langle x, x\rangle & -x^{\mathbf{T}} B_{1} & 1-\frac{1}{2}\langle x, x\rangle
\end{array}\right]
\end{gathered}
$$

Note that one can also obtain the last expression for $n(x)$ from $n(x)=$ $\Theta(v(x))=J v(x) J$.

The Bruhat decomposition allows one to characterize the homogeneous space $G / A \tilde{M}_{1} W_{+}$and, as will be shown in the next section, to obtain explicit formulas for the group action. We now work out this characterization.

Let $\mathscr{P}^{n+2}$ denote projective $(n+2)$-space. This is realized as the set of equivalence classes $\{\lambda x: \lambda \in \mathbb{R}, \lambda \neq 0\}$ of non-zero vectors $x$ in $\mathbb{R}^{n+3}$. For $0 \neq x \in \mathbb{R}^{n+3}$ let $[x]$ denote the corresponding element in $\mathscr{P}^{n+2}$. One defines an action of the general linear group $\mathrm{Gl}(n+3, \mathbb{R})$ on the projective space $\mathscr{P}^{n+2}$ by $g[x]=[g x]$, where $g \in \mathrm{Gl}(n+3, \mathbb{R})$, $x \in \mathbb{R}^{n+3}$, and $(g, x) \Rightarrow g x$ is the usual action of matrices on $\mathbb{R}^{n+3}$.

We look at the orbit of the vector $\left(1,0_{n+1}, 1\right)^{\mathbf{T}}$ in $\mathbb{R}^{n+3}$, and also the point $\left[\left(1,0_{n+1}, 1\right)^{\mathrm{T}}\right]$ in $\mathscr{P}^{n+2}$. One sees from (3.7) that the vector $\left(1,0_{n+1}, 1\right)^{\mathrm{T}}$ is fixed by the subgroup $W_{+}$. This vector is also fixed by the subgroup $M_{1}$. The element $B$ takes this vector to the vector $-\left(1,0_{n+1}, 1\right)^{\mathbf{T}}$. Thus from Proposition 3 the elements of the first double coset in the Bruhat decomposition (Proposition 5) map this vector into

$$
\left( \pm e^{t}(1+\langle x, x\rangle), 2 e^{t} x, \pm e^{t}(1-\langle x, x\rangle)\right)^{\mathbf{T}}, \quad x \in \mathbb{R}^{n+1},
$$

while the elements of the second double coset map this vector into

$$
\left(\mp e^{t}, 0_{n+1}, \pm e^{t}\right)^{\mathbf{T}} \text {. }
$$

These considerations lead immediately to the following lemma:

Lemma 5. Suppose $g \in G$. Write

$$
g\left(\begin{array}{c}
1 \\
0^{n} \\
1
\end{array}\right)=\left(\begin{array}{l}
\alpha \\
\beta \\
\gamma
\end{array}\right) .
$$


Then $\alpha+\gamma= \pm 2 e^{t}, \alpha-\gamma= \pm 2 e^{t}\langle x, x\rangle$ and $\beta=2 e^{t} x$, for $g$ in the first double coset of the Bruhat decomposition with $g=v(x) a(t) m n(y)$, $x \in \mathbb{R}^{n+1}$, and $m \in \tilde{M}_{1}$. The + sign occurs when $m$ is in the identity component of $\tilde{M}$, and the minus sign otherwise. For $g$ in the second double coset one has $\beta=0$, and $\alpha+\gamma=0, \alpha-\gamma=\mp 2 e^{t}$.

One also notes:

Proposition 6. The orbit in $\mathscr{P}^{n+2}$ containing the point $\left[\left(1,0_{n+1}, 1\right)^{\mathrm{T}}\right]$ is

$$
\left\{\left[\left(\frac{1}{2}(1+\langle x, x\rangle), x, \frac{1}{2}(1-\langle x, x\rangle)\right)^{\mathbf{T}}\right]: x \in \mathbb{R}^{n+1}\right\} \cup\left\{\left[\left(-1,0_{n+1}, 1\right)^{\mathbf{T}}\right]\right\} .
$$

The isotropy subgroup is $A \tilde{M}_{1} W_{+}$.

Proof. One applies Lemma 5 and the formulas (3.6) and (3.7). The statement then follows from the Bruhat decomposition.

Thus, this orbit may be identified with the coset space $G / A \tilde{M}_{1} W_{+}$. For $t=0$, the elements in the first column second row of the matrix (3.6) is just $x$. Hence the map $x \mapsto v(x)$ is a one to one map from $\mathbb{R}^{n+1}$ to $W_{-}$. Hence, all the points in this orbit, with the exception of the point $\left[\left(-1,0_{n+1}, 1\right)^{\mathbf{T}}\right]$, are coordinatized by $\mathbb{R}^{n+1}$. Denote the exceptional point by $\infty$. The non-exceptional points will be referred to as finite points.

It follows from the Bruhat decomposition that in computing the action of $G$ it is sufficient to consider the action of the subgroups $W_{-}$(translations), $A$ (dilatations), $\tilde{M}_{1}$ (orthogonal transformations or homogeneous Lorentz transformations) and $W_{+}$(the conjugate translations).

Write for $g \in G$,

$$
g=\left(\begin{array}{lll}
A_{11} & A_{12} & A_{13} \\
A_{21} & A_{22} & A_{23} \\
A_{31} & A_{32} & A_{33}
\end{array}\right),
$$

for $A_{11}, A_{13}, A_{31}, A_{33} \in \mathbb{R}, A_{12}, A_{32} \in \mathbb{R}_{n+1}, A_{21}, A_{23} \in \mathbb{R}^{n+1}, A \in \mathbb{R}_{n+1}^{n+1}$. Then it follows again from Lemma 4 and formulas (3.6) and (3.7) that the point $x$ corresponding to $\left[g\left(1,0_{n+1}, 1\right)^{\mathbf{T}}\right]=\left[(\alpha, \beta, \gamma)^{\mathbf{T}}\right]$ is given by

$$
x=\left(A_{21}+A_{23}\right) /\left(A_{11}+A_{13}\right)=\beta /(\alpha+\gamma),
$$

with a zero denominator interpreted as the point $\infty$. This observation follows from Lemma 4. 
Thus if we write the action of $g$ in the above coordinates as $x^{\prime}=g \cdot x$, then

$$
\begin{aligned}
& x^{\prime}=x+a, \quad \text { if } g=v(a), \quad x^{\prime}=e^{t} x, \quad \text { if } g=\exp t H, \\
& x^{\prime}=A_{22} x, \quad g \in \tilde{M}, \text { usual linear action of } A_{22} \text { for } x \in \mathbb{R}^{n+1}, \\
& A_{22} \infty=\infty, \\
& x^{\prime}=\frac{x-\langle x, x\rangle}{1+\langle a, a\rangle\langle x, x\rangle-2\langle x, a\rangle}, \quad g=n(a) .
\end{aligned}
$$

REMARK 1. Formula 3.10 implies that the orbit of $G$ in $\mathscr{P}^{n+2}$ can be identified with the projective space $\mathscr{P}^{n+1}$.

Let $\tilde{G}$ be the group generated by the element $J$ and the group $G$. Recall that $J=\operatorname{diag}[-1,1,1, \ldots, 1]$. Thus $\tilde{G}$ is a subgroup of $\mathrm{O}(2, n+1)$, and contains $\mathrm{SO}_{e}(2, n+1)$. It follows from statement 2 of the last proposition that one has:

$$
J \cdot x=\frac{x}{\langle x, x\rangle},
$$

for $x$ a finite point, and $J \cdot \infty=0^{n+1}$. This mapping may be interpreted as the reflection in the unit quadric defined by $\langle x, x\rangle=1$. From the last proposition one obtains the following proposition as a corollary:

Proposition 7. The group $\tilde{G}$ is generated as a transformation group on the projective space by the orthogonal transformations $M_{1}$, the translations $W_{-}=\left\{v(a): a \in \mathbb{R}^{n}\right\}$, the dilatations, and the inversion $x \mapsto$ $J \cdot x$.

Proof. This statement follows from Proposition 6, statement 3 and the observation that

$$
\begin{aligned}
(J v(-a) J) \cdot x & =J \cdot \frac{x-a\langle x, x\rangle}{\langle x, x\rangle} \\
& =\frac{x-a\langle x, x\rangle}{1-2\langle a, x\rangle+\langle a, a\rangle\langle x, x\rangle}=n(a) \cdot x .
\end{aligned}
$$

REMARK 2. In case the form $\langle$,$\rangle is positive definite, the transfor-$ mations of the projective space $\mathscr{P}^{n+1}$ corresponding to the group $\tilde{G}$ are called the Möbius transformations. The characterization in Proposition 7 is usually taken to be the definition of this group.

REMARK 3. Since $J W_{-} J=\Theta W_{-}=W_{+}, \tilde{G}$ is generated by the subgroups $\tilde{M}_{1}, W_{-}, A$, and the elements of $C$ and $J$. The above proposition could also be deduced from this fact. However it is useful to have the above explicit formulas for the action of $\tilde{G}$ on $\mathscr{P}^{n+1}$. 
4. Infinitesimal actions for the Lorentz groups. From now on we write $W=W_{+}$and $P=P_{+}$. For every complex number $l$ there exists a homomorphism $\lambda$ of the subgroup $A$ onto the multiplicativle group of the non-zero complex numbers $\mathbb{C}^{*}$ defined by $\lambda(\exp (t H))=e^{t l}$. Let $\lambda$ be such a homomorphism. We extend this to a mapping from the entire subgroup $P$ to $\mathbb{C}^{*}$ by writing $\lambda(a m w)=\lambda(a)$, with $m \in \tilde{M}_{1}$, and $w \in W$. However this extended map is in fact a homomorphism as one easily checks from the fact that $\tilde{M}_{1} W$ is a normal subgroup of $P$. The set of such homomorphisms, called pseudo-characters of $P$, is denoted by $\tilde{\mathbb{C}}$.

For $\lambda \in \tilde{\mathbb{C}}$, let $C_{\lambda}^{\infty}(G)$ be space of complex valued functions having continuous derivatives up to arbitrary order, and having the transformation properties:

$$
f(g p)=\lambda\left(p^{-1}\right) f(g), \quad p \in P, f \in C_{\lambda}^{\infty}(G) .
$$

Let $\mathscr{S}\left(\mathbb{R}^{n+1}\right)$ be the Schwartz space of rapidly decreasing functions on $\mathbb{R}^{n+1}$. Recall that the map $v$ from $\mathbb{R}^{n+1}$ to $W_{-}$defined by (3.5) of the previous section is a diffeomorphism. Let $\mathscr{S}\left(W_{-}\right)$denote the space of functions on $W_{-}$defined by

$$
\mathscr{S}\left(W_{-}\right)=\left\{f \circ v^{-1}: f \in \mathscr{S}\left(\mathbb{R}^{n+1}\right)\right\} .
$$

Finally let $\lambda \in \tilde{\mathbb{C}}$. By the Bruhat decomposition define the space $\mathscr{S}_{\lambda}(G)$ of the functions $f$ on $G$ as follows:

$$
\begin{aligned}
f(w p) & =\lambda\left(p^{-1}\right) f(w), \quad w \in W_{-}, p \in P, f \in \mathscr{S}\left(W_{-}\right), \\
& =0 \text { on } B P .
\end{aligned}
$$

LEMMA 6. One has the inclusion $\mathscr{S}_{\lambda}(G) \subset C_{\lambda}^{\infty}(G)$.

Proof. The point is that the functions in $\mathscr{S}_{\lambda}(G)$ together with their derivatives are continuous at the coset $C \tilde{M}_{1} A W_{+}$. However, in accordance with the discussion of the previous section this coset corresponds to the limit point $\infty$ in $\mathscr{P}^{n+1}$. The definition of $\mathscr{S}\left(\mathbb{R}^{n+1}\right)$ implies that the functions in this space and their derivatives have the limit 0 as $|\langle x, x\rangle| \rightarrow \infty$ in $\mathbb{R}^{n+1}$. Since the functions in $\mathscr{S}_{\lambda}(G)$ are determined by their restrictions to $W_{-}$, the result follows.

The group $G$ acts on $\mathscr{S}_{\lambda}(G)$ by left translations: $L(g) f(h)=$ $f\left(g^{-1}(h), g, h \in G, f \in \mathscr{S}_{\lambda}(G)\right.$. For a fixed pseudo-character $\lambda$, let $\Pi_{\lambda}$ denote the transfer of this action to the Schwartz space $\mathscr{S}\left(\mathbb{R}^{n+1}\right)$; that is, $\Pi_{\lambda}(g)=\rho L(g) \rho^{-1}$, where $\rho$ is the map from $\mathscr{S}_{\lambda}(G)$ to $\mathscr{S}\left(\mathbb{R}^{n+1}\right)$ 
defined by $(\rho f)(x)=(f \circ v)(x), x \in \mathbb{R}^{n+1}$. (This map is a linear isomorphism, by the above discussion.) For $X \in \mathbb{G}$, the Lie algebra of $G$, one defines the operator $d \Pi_{\lambda}(X)$ by

$$
\begin{aligned}
d \Pi_{\lambda}(X) \rho f(x) & =\left.\frac{d}{d t} \Pi_{\lambda}(\exp (t X)) \rho f(x)\right|_{t=0} \\
& =\left.\rho \frac{d}{d t} f(\exp (-t X) v(x))\right|_{t=0} .
\end{aligned}
$$

Then $X \rightarrow d \Pi_{\lambda}(X)$ is a representation (homomorphism) of the Lie algebra $G$ into the Lie algebra of differential operators defined on the space $\mathscr{S}\left(\mathbb{R}^{n+1}\right)$. The first task is to describe this Lie algebra action explicitly. From the Lie algebra Bruhat decomposition Lemma 1, it is sufficient to look at the actions of the subalgebras $A, M_{1}, V_{-}$, and $W_{+}$. The operators $p(x) \cdot \nabla$ for $x \rightarrow p(x)$ a $C^{\infty}$-self mapping of $\mathbb{R}^{n+1}$ is defined in a manner analogous to the definition of $\S 2$. The Lie algebra action is given in the following statement:

Proposition 8. For $a \in \mathbb{R}^{n+1}$, and for $T \in \mathbf{M}$.

$$
\begin{aligned}
d \Pi_{\lambda}(V(a)) & =-a \cdot \nabla, \\
d \Pi_{\lambda}(N(a)) & =-2\langle a, x\rangle l+\langle x, x\rangle a \cdot \nabla-2\langle x, a\rangle x \cdot \nabla, \\
d \Pi_{\lambda}(H) & =(x \cdot \nabla+l \mathbf{1}) \\
d \Pi_{\lambda}(T) & =-\mathbf{T}(x) \cdot \nabla,
\end{aligned}
$$

where $T$ and $\mathbf{T}$ are defined by

$$
T=\left[\begin{array}{ccc}
0 & 0_{n+1} & 0 \\
0^{n+1} & \mathbf{T} & 0^{n+1} \\
0 & 0_{n+1} & 0
\end{array}\right]
$$

$\mathbf{T} \in \operatorname{so}(1, n)$.

ReMARK 1. Write for $b \in \mathbb{R}^{n+1}, b^{t}=b^{T} B_{1}$. If $a, b \in \mathbb{R}^{n+1}$ then $a b^{t}-b a^{t}$ is an element of the Lie algebra so $(1, n)$. By definition, this is the Lie algebra of the orthogonal group of the form $(x, y) \Rightarrow$ $\langle x, y\rangle=x^{t} y=x B_{1} y$. It is known that this Lie algebra is spanned by such elements. Recall then that subalgebra $M_{1}=\operatorname{diag}[1, \operatorname{so}(1, n), 1]$.

Proof of the proposition. For the first statement recall that $v(a) v(x)$ $=v(a+x)$. Thus by definition of the action (4.1) one has for $f \in$ $\mathscr{S}\left(\mathbb{R}^{n+1}\right)$, recalling that $v(x)=\exp (V(x))$,

$$
d \Pi_{\lambda}(V(a))=\left.\frac{d}{d t} f(x-t a)\right|_{t=0}=-a \cdot \nabla f(x) .
$$


Observe in general for $Z \in M_{1} \oplus A \oplus \mathbb{N}$,

$$
Z v(x)=Z \exp (V(x))=\exp (V(x)) \exp [\operatorname{ad}(-V(x))] Z=v(x) Z^{\prime},
$$

where

$$
Z^{\prime}=\sum_{j=0}^{\infty} \frac{1}{j !}(-\operatorname{ad} V(x))^{j} Z .
$$

Therefore writing for $f \in \mathscr{S}\left(\mathbb{R}^{n+1}\right), \phi=\rho^{-1} \circ f$

$$
\begin{aligned}
d \Pi_{\lambda}(Z) f(x) & =\left.\frac{d}{d t} \phi(\exp (-t Z) v(x))\right|_{t=0} \\
& =\left.\frac{d}{d t} \phi\left(v(x) \exp \left(-t Z^{\prime}\right)\right)\right|_{t=0} .
\end{aligned}
$$

Note that $(\operatorname{ad}(V(x)))^{j} Z=0$ if $j \geq 3$. This remark follows from the fact the eigenvalues of $\operatorname{ad}(H)$ are $-1,0$, and 1 . In case $Z=N(a), Z^{\prime}$ becomes

$$
\begin{aligned}
Z^{\prime} & =\exp (\operatorname{ad}(-V(x)) N(a)) \\
& =N(a)-[V(x), N(a)]+\frac{1}{2}[V(x),[V(x), N(a)]] \\
& =N(a)+2\left(a x^{t}-x a^{t}\right) E_{22}-2\langle x, a\rangle H-\langle x, x\rangle V(a)+2\langle x, a\rangle V(x),
\end{aligned}
$$

where we write

$$
\left(a x^{t}-x a^{t}\right) E_{22}=\left[\begin{array}{ccc}
0 & 0_{n+1} & 0 \\
0^{n+1} & a x^{t}-x a^{t} & 0^{n+1} \\
0 & 0_{n+1} & 0
\end{array}\right] .
$$

The second formula follows from 4.2. For the third formula, set $Z=$ $H$. Then $Z^{\prime}$ becomes

$$
Z^{\prime}=H-[V(x), H]=H-V(x) .
$$

Thus the third result follows again from (4.2). Finally setting $Z=$ $T \in \mathrm{M}_{1}$, then $Z^{\prime}=T-[V(x), T]=T+V(T x)$, as one easily computes. Thus the last equation follows.

REMARK 2. One knows from general principles that the operators given in the above proposition provide a representation of the Lie algebra $\mathbb{G}$. However, it is interesting to check the commutation relations directly. This can be accomplished by observing as in (2.1) with $n$ replaced by $n+1$,

$$
[p(x) \cdot \nabla, q(x) \cdot \nabla]=(p(x) \cdot \nabla q(x)) \cdot \nabla-(q(x) \cdot \nabla p(x)) \cdot \nabla .
$$


Thus for example one obtains

$$
\begin{aligned}
& {\left[d \Pi_{\lambda}(V(b)), d \Pi_{\lambda}(N(a))\right]} \\
& \quad=-\langle x, b\rangle a \cdot \nabla+\langle x, a\rangle b \cdot \nabla+2\langle a, b\rangle(x \cdot \nabla+l 1) .
\end{aligned}
$$

Notice that this says that if $a$ is orthogonal to $b$, then this commutator is $2 \Pi_{\lambda}(T)$ with $T$ corresponding to the element $\mathbf{T}=a b^{t}-b a^{t}$. If $a=b$, then the commutator is a generator of the one dimensional algebra $d \Pi_{\lambda}(\mathrm{A})$.

Let $\left\{e_{i}: 0 \leq i \leq n\right\}$ be the natural basis $\mathbb{R}^{n+1}$. Thus, $\left\langle e_{i}, e_{j}\right\rangle=0$, if $i \neq j$, and $\left\langle e_{i}, e_{i}\right\rangle=\varepsilon_{i}$, where $\varepsilon_{i}=-1$, if $i=0$, and $\varepsilon_{i}=1$, if $1 \leq i \leq n$. Then note that $\left\langle x, e_{i}\right\rangle=\left\langle e_{i}, x\right\rangle=\varepsilon_{i} x_{i}$, and $e_{i} \cdot \nabla=\partial_{i}:=\partial / \partial x_{i}$. One has in terms of the basis introduced in $\S 2$,

$$
\begin{aligned}
& V\left(e_{0}\right)=Y+X, \quad V\left(e_{i}\right)=Y_{i}+X_{i}, \quad \text { for } 1 \leq i \leq n . \\
& N\left(e_{0}\right)=Y-X, \quad N\left(e_{i}\right)=-Y_{i}+X_{i}, \quad \text { for } 1 \leq i \leq n .
\end{aligned}
$$

Define the elements $V_{i}$ and $N_{i}$ by

$$
V_{i}=\frac{1}{2} V\left(e_{i}\right), \quad N_{i}=\frac{1}{2} N\left(e_{i}\right), \quad 0 \leq i \leq n .
$$

To simplify the notation write

$$
\begin{aligned}
\mathbf{N}_{j} & =\frac{1}{2} d \Pi_{\lambda}\left(N\left(e_{i}\right)\right)=d \Pi_{\lambda}\left(N_{i}\right), \\
\mathbf{V}_{i} & =d \Pi\left(V_{i}\right)=\frac{1}{2} d \Pi_{\lambda}\left(V\left(e_{i}\right)\right), \\
\mathbf{H} & =d \Pi_{\lambda}(H), \quad \text { and } \quad \mathbf{L}_{i j}=d \Pi_{\lambda}(T) \quad \text { for } T=\left(e_{i} e_{j}^{t}-e_{j} e_{i}^{t}\right) E_{22} .
\end{aligned}
$$

(This notation is consistent with that used in §2.) In fact, one computes

$$
\left(e_{i} e_{j}^{t}-e_{j} e_{i}^{t}\right) x=e_{i}\left\langle e_{j}, x\right\rangle-e_{j}\left\langle e_{i}, x\right\rangle=X_{i j} x,
$$

if $1 \leq i<j \leq n$, and

$$
\begin{gathered}
\left(e_{0} e_{j}^{t}-e_{j} e_{0}^{t}\right) x=\left(e_{0} e_{j}^{\mathbf{T}}+e_{j} e_{0}^{\mathbf{T}}\right) x=Z_{j} x . \\
\mathbf{N}_{i}=\frac{1}{2}\langle x, x\rangle \partial_{i}-\varepsilon_{i} x_{i}(E+l \mathbf{1}), \quad \varepsilon_{i}=1, i \geq 1, \quad \varepsilon_{0}=-1 . \\
\quad \mathbf{V}_{i}=-\frac{1}{2} \partial_{i}, \quad i=0,1, \ldots, n, \\
\mathbf{H}=(E+l 1), \\
\mathbf{L}_{i j}=\varepsilon_{i} x_{i} \partial_{j}-\varepsilon_{j} x_{j} \partial_{i} \\
=x_{i} \partial_{j}-x_{j} \partial_{i}, \quad \text { if } 1 \leq i<j \leq n, \\
=-\left(x_{0} \partial_{j}+x_{j} \partial_{0}\right), \quad \text { if } i=0<j \leq n .
\end{gathered}
$$

REMARK 2 (continued). Again, pursuing the comment of Remark 2 one may check directly that,

$$
\left[\mathbf{V}_{i}, \mathbf{N}_{j}\right]=-\frac{1}{2}\left\{\varepsilon_{i} x_{i} \partial_{j}-x_{j} \varepsilon_{j} \partial_{i}\right\}
$$


for $i \neq j$, and

$$
\left[\mathbf{V}_{i}, \mathbf{N}_{i}\right]=\frac{1}{2}(E+\mathbf{1} l) \varepsilon_{i}=\frac{1}{2} \mathbf{H} \varepsilon_{i}
$$

5. Some elements of the universal enveloping algebra. For a Lie algebra $\mathbb{L}$ let $U(\mathbb{L})$ denote the universal enveloping algebra of $\mathbb{L}$. Recall that by definition this is the quotient algebra of the tensor algebra $T(\mathbb{L})$ modulo the ideal $\mathscr{I}$ generated by the elements $X \otimes Y-Y \otimes X-$ $[X, Y]$ for $X, Y \in \mathbb{L}$. This algebra is characterized by the following universality property:

Let $\varphi$ be a homomorphism of the Lie algebra $\mathbb{L}$ into some associative algebra $\mathscr{A}$, with Lie multiplication [, ] on $\mathbb{L}$ going over into commutators in $\mathscr{A}$. Then $\varphi$ extends uniquely to a homomorphism $\varphi$ of the associative algebra $U(\mathbb{L})$ into the associative algebra $\mathscr{A}$.

Thus, in the setting of the previous section, for each pseudo-character $\lambda$ the Lie algebra representation $d \Pi_{\lambda}$ of $\mathbb{G}$ extends to a homomorphism, also denoted by $d \Pi_{\lambda}$, of $U(\mathbb{G})$ into the algebra of differential operators on $\mathscr{S}\left(\mathbb{R}^{n+1}\right)$. The image of this homomorphism is just the subalgebra consisting of the differential operators generated by the elements $d \Pi_{\lambda}(X), X \in \mathbb{G}$.

Remarks. Some additional remarks are needed concerning the extension of derivations and automorphisms of Lie algebras to their universal enveloping algebras. Let $A$ be an automorphism of a Lie algebra $\mathrm{L}$. Then by definition, $A$ is a linear map on $\mathrm{L}$ such that $A(X, Y)=[A(X), A(Y)] . A$ extends to an automorphism of the tensor algebra $T(\mathbb{L})$ such that

$$
A\left(x_{1} \otimes x_{2} \otimes \cdots \otimes x_{n}\right)=A x_{1} \otimes A x_{2} \otimes \cdots \otimes A x_{n},
$$

for $x_{i} \in \mathbb{L}$. It follows from the fact $A$ is an automorphism of the Lie algebra that the ideal $\mathscr{I}$ is invariant under $A$. Hence $A$ induces an automorphism on the universal enveloping algebra $U(\mathbb{L})$, also denoted by the same symbol $A$.

We apply this extension of automorphisms to groups acting on the Lie algebras. If $\tilde{L}$ is a Lie group (not necessarily connected) whose Lie algebra is $\mathbb{L}$, then $\tilde{L}$ acts on $\mathbb{L}$ as automorphisms by $g \Rightarrow \operatorname{Ad}(g)$. By the above considerations this action extends to the universal enveloping algebra $U(\mathbb{L})$. Let $L$ denote the identity component of $\tilde{L}$. The differential of the action Ad of $L$ is just the extension of the inner derivations ad on $U(\mathbb{L})$. More explicitly one has:

$$
\operatorname{ad}(X) q=\left.\frac{d}{d t} \operatorname{Ad}(\exp t X) q\right|_{t=0}
$$

for $X \in \mathbb{L}$, and $q \in U(\mathbb{L})$. Thus if $q \in U(\mathbb{L})$, then $\operatorname{ad}(X) q=[X, q]$. 
Now if $\mathbb{L}$ is any subalgebra of $\mathbb{G}$, it follows from universality that we may identify $U(\mathbb{L})$ with the subalgebra of $U(\mathbb{G})$ generated by the elements of $L$. We do in fact make these identifications. We have the following definition:

Definition. Let $\tilde{L}$ be a Lie subgroup of $G$ (not necessarily connected). An element $q \in U(\mathbb{L})$ is said to be $\tilde{L}$ invariant if $\operatorname{Ad}(h) q=q$ for all $h \in \tilde{L}$.

It follows from the above remarks that if an element $q$ is $L$ invariant for $L$ the identity component of $\tilde{L}$, then this condition is equivalent to $\operatorname{ad}(X) q=0$ for all $X \in \mathbb{L}$.

Let $\langle$,$\rangle denote the bilinear form on \mathbb{G}$ defined by $\langle X, Y\rangle=$ $-\frac{1}{2} \operatorname{Trace}\left(X, Y^{\mathbf{T}}\right)$, for $X, Y \in \mathbb{G}$. This bilinear form has the following properties:

(i) $\langle$,$\rangle is symmetric and positive definite.$

(ii) $\langle$,$\rangle is invariant in the sense that \langle[X, Y], Z\rangle=\left\langle Y,\left[X^{\mathbf{T}}, Z\right]\right\rangle$, all $X, Y, Z \in \mathbb{G}$. In other terms the adjoints of the operators $\operatorname{ad}(X)$ with respect to the form $\langle$,$\rangle are given in terms of transposition by$ $\operatorname{ad}(X)^{*}=\operatorname{ad}\left(X^{\mathbf{T}}\right)$. These properties are easily checked from the involution property of transposition, and the standard properties of the trace.

Let $\left\{\mathbf{U}_{k}\right\}$ be any orthonormal basis of $\mathbb{G}$ relative to the form $\langle$, define the Casimir element of $U(\mathbb{G})$ to be

$$
\Omega_{G}=-\sum_{j=-1}^{n+1} \mathbf{U}_{j} \mathbf{U}_{j}^{\mathbf{T}} .
$$

Of course multiplication is in the universal enveloping algebra. More generally, if $\mathbb{H}$ is any reductive (direct sum of semisimple and abelian) subalgebra of $\mathbb{G}$, and $\left\{V_{k}\right\}$ is an orthonormal basis of $\mathbb{H}$, then one defines the Casimir element of $H$ as $\Omega_{H}=-\sum V_{k} V_{k}^{\mathbf{T}}$. The following facts are standard and not proved here:

(i) For $\mathrm{H}$ a reductive Lie subalgebra the definition of $\Omega_{H}$ is independent of the orthonormal basis chosen.

(ii) The element $\Omega_{H}$ commutes with all the elements of $\mathbb{H}$, and hence with the elements of $U(\mathbb{H})$. In particular $\Omega_{G}$ is in the center of the universal enveloping algebra of $\mathbb{G}$.

Remarks. (i) The definitions of the Casimir elements differ from those usually given for semisimple Lie algebras in so far as the form $\langle$,$\rangle replaces the Killing forms. A Schur lemma argument shows that$ 
the elements defined here differ from the usual ones by a real constant. Our definitions have the advantage that by using a standardized invariant form the sum formulas given below take a simpler form.

(ii) The choice of sign guarantees that if $H$ is a compact subalgebra, then the eigenvalues of $\Omega_{H}$ are positive.

Note that the basis introduced in $\S 2$ is indeed an orthonormal basis with respect to the form $\langle$,$\rangle . Also X^{\mathbf{T}}=X$ for $X$ symmetric, and $X^{\mathbf{T}}=-X$ for $X$ skew. One has the following:

$$
\Omega_{M}=-\sum_{i \geq 1}^{n-1} \sum_{j>i}^{n} X_{i j}^{2} .
$$

If one defines the elements $\mathscr{Z}, \mathscr{Y}$, and $\mathscr{Z}$ by

$$
\mathscr{Z}=\sum_{k=1}^{n} Z_{k}^{2}, \quad \mathscr{Y}=\sum_{k=1}^{n} Y_{k}^{2}, \quad \mathscr{X}=-\sum_{k=1}^{n} X_{k}^{2},
$$

then one has from the foregoing remark and from the definitions of the algebras $M_{1}, M_{2}$, and $M_{3}$ in $\S 3$,

$$
\begin{aligned}
\Omega_{M_{1}} & =\mathscr{Z}+\Omega_{M}, \quad \Omega_{M_{2}}=\mathscr{Y}+\Omega_{M}, \quad \Omega_{M_{3}}=\mathscr{X}+\Omega_{M} . \\
\Omega_{K} & =Y^{2}+\mathscr{Z}+\mathscr{X}+\Omega_{M}=Y^{2}+\mathscr{X}+\Omega_{M_{1}}=Y^{2}+\mathscr{Z}+\Omega_{M_{3}} .
\end{aligned}
$$

It is useful to define the element $\Omega_{L} \in U(\mathbb{G})$ by

$$
\Omega_{L}=\mathscr{Y}+H^{2}-X^{2} \text {. }
$$

Then from (5.1),

$$
\Omega_{G}=H^{2}-X_{2}^{2}+\mathscr{Y}+\Omega_{K}=\Omega_{L}+\Omega_{K} .
$$

We shall also need to express the elements $\Omega_{K}$ and $\Omega_{G}$ in terms of the bases of $W_{+}$and $W_{-}$introduced in $\S 3$, and more explicitly in (4.4). Note first that from (4.4) and (5.2)

$$
\mathscr{Z}+Y^{2}=-\sum_{k=1}^{n}\left(V_{k}+N_{k}\right)^{2}+\left(V_{0}+N_{0}\right)^{2} .
$$

By using the commutation rules $-\left[N_{0}, V_{0}\right]=\frac{1}{2} H=\left[N_{k}, V_{k}\right]$, one gets the following two expressions:

$$
\begin{aligned}
\mathscr{X}+Y^{2} & =-\sum_{k \geq 0}^{n} \varepsilon_{k}\left(V_{k}^{2}+N_{k}^{2}\right)-2 \sum_{k \geq 0}^{n} \varepsilon_{k} V_{k} N_{k}+\frac{1}{2}(n+1) H \\
& =-\sum_{k \geq 0}^{n} \varepsilon_{k}\left(V_{k}^{2}+N_{k}^{2}\right)-2 \sum_{k \geq 0}^{n} \varepsilon_{k} N_{k} V_{k}-\frac{1}{2}(n+1) H .
\end{aligned}
$$


Recall that $\varepsilon_{k}=1$, if $k \geq 1$, and $\varepsilon_{0}=-1$. Similarly,

$$
\begin{aligned}
\Omega_{L} & =\mathscr{Y}+H^{2}-X^{2}=\sum_{k \geq 1}^{n}\left(V_{k}-N_{k}\right)^{2}-\left(V_{0}-N_{0}\right)^{2}+H^{2} \\
& =\sum_{k \geq 0}^{n} \varepsilon_{k}\left(V_{k}^{2}+N_{k}^{2}\right)-2 \sum_{k \geq 0}^{n} \varepsilon_{k} V_{k} N_{k}+\frac{1}{2}(n+1) H+H^{2} \\
& =\sum_{k \geq 0}^{n} \varepsilon_{k}\left(V_{k}^{2}+N_{k}^{2}\right)-2 \sum_{k \geq 0}^{n} \varepsilon_{k} N_{k} V_{k}-\frac{1}{2}(n+1) H+H^{2}
\end{aligned}
$$

In particular, from the second expression in (5.3), (5.5a), and (5.5b),

$$
\begin{aligned}
\Omega_{K} & =-\sum_{k \geq 0}^{n} \varepsilon_{k}\left(V_{k}^{2}+N_{k}^{2}\right)-2 \sum_{k \geq 0}^{n} \varepsilon_{k} N_{k} V_{k}-\frac{1}{2}(n+1) H+\Omega_{M_{1}} \\
& =-\sum_{k \geq 0}^{n} \varepsilon_{k}\left(V_{k}^{2}+N_{k}^{2}\right)-2 \sum_{k \geq 0}^{n} \varepsilon_{k} V_{k} N_{k}+\frac{1}{2}(n+1) H+\Omega_{M_{1}} .
\end{aligned}
$$

From (5.4), (5.6a), and (5.7b),

$$
\Omega_{G}=H^{2}+(n+1) H-4 \sum_{k \geq 0}^{n} \varepsilon_{k} V_{k} N_{k}+\Omega_{M_{1}} .
$$

Proposition 9. The elements $\Omega_{L}$ and $\Omega_{K}$ are invariant under the subgroup $\tilde{K}$ and under the Lie algebra $\mathbb{K}$.

Proof. By the above remarks $\Omega_{G}$ is invariant under $\mathbb{G}$, and therefore under the subalgebra $\mathbb{K}$. Since $\Omega_{K}$ is the Casimir element of the latter subalgebra it is also invariant under $\mathbb{K}$. Therefore, since $\Omega_{L}=\Omega_{G}-$ $\Omega_{K}, \Omega_{L}$ is also invariant under $\mathbb{K}$. This assertion is equivalent to the assertion that $\Omega_{L}$ is invariant under the connected subgroup $K$. By the coset decomposition (3.1), it remains to check that this element is invariant under the automorphism $X \hookrightarrow B X B$ of the Lie algebra $\mathbb{G}$. However, by one of the defining relations for the Lie algebra $\mathbb{G}$, $B Y B=Y^{\mathrm{T}}$, for $Y \in \mathbb{G}$. Hence, if $Y$ is either a symmetric or a skew symmetric element of $\mathbb{G}$, then the tensor product $Y \otimes Y$ is invariant under this automorphism. Therefore the image modulo $\mathscr{I}$ is invariant under this automorphism. It follows that all the elements defined in (5.2), (5.3) and (5.4) are invariant under this automorphism. 
6. Invariant differential operators. Next we compute the representations of the elements of the universal enveloping algebra of $\mathbb{G}$ in the representation $d \Pi_{\lambda}$. Define the wave operator by

$$
\square=\sum_{k=0}^{n} \partial_{k}^{2}-\partial_{0}^{2} .
$$

THEOREM 2. Let $\lambda$ be a pseudocharacter of the subgroup $P$ with $\mathbb{B}$ the complex number defined by $\lambda(\exp t H)=e^{l t}$. Then in the representation $d \Pi_{\lambda}$ one has:

$$
\begin{aligned}
d \Pi_{\lambda}\left(\Omega_{G}\right)= & l\left(l-\frac{1}{2}(n+1)\right) \mathbf{1} \\
d \Pi_{\lambda}\left(\Omega_{L}\right)= & \frac{1}{4}(1+\langle x, x\rangle)^{2} \square+\left(l-\frac{1}{2}(n-1)\right)(1+\langle x, x\rangle)(E+l \mathbf{1})-l \mathbf{1} \\
d \Pi_{\lambda}\left(\Omega_{K}\right)= & -\frac{1}{4}(1+\langle x, x\rangle)^{2} \square \\
& -\left(l-\frac{1}{2}(n-1)\right)\{(1+\langle x, x\rangle)(E+l \mathbf{1})-l \mathbf{1}\} \\
d \Pi_{\lambda}\left(\Omega_{M_{1}}\right)= & E^{2}+(n-1) E-\langle x, x\rangle \square .
\end{aligned}
$$

Proof. Using the notation of $\S 4$, let $f \in \mathscr{S}\left(\mathbb{R}^{n+1}\right), \rho^{-1} f \in \mathscr{S}_{\lambda}(G)$. Since the latter space consists of $C^{\infty}$-functions on the group $G$, (Lemma 6) it makes sense to consider the representation $R$ of $U(\mathbb{G})$ defined by right translations. However, since $\Omega_{G}$ is in the center of the enveloping algebra and since $G$ is connected, $L(g) d L\left(\Omega_{G}\right)=$ $d L\left(\Omega_{G}\right) L(g)$ all $g \in G$. Moreover for $\varphi$ a differentiable function on $G$ one has at the identity $e \in G: R\left(\Omega_{G}\right) \varphi(e)=L\left(\Omega_{G}\right) \varphi(e)$. With these preliminary remarks one gets for $f \in \mathscr{S}\left(\mathbb{R}^{n+1}\right), x \in \mathbb{R}^{n+1}$ from equation (5.8), by writing $\varphi=\rho^{-1}$ :

$$
\begin{gathered}
d \Pi_{\lambda}\left(\Omega_{G}\right) f(x)=\rho L\left(\Omega_{G}\right) \varphi(v(x))=\rho L\left(\Omega_{G}\right) L(v(x)) \varphi(e) \\
\quad=\rho L(v(x)) L\left(\Omega_{G}\right) \varphi(e)=\rho L(v(x)) R\left(\Omega_{G}\right) \varphi(e) \\
=\rho L(v(x))\left(\left[(-\lambda(H))^{2}+\frac{1}{2}(n+1)(-\lambda(H))+\Lambda\left(\Omega_{M_{1}}\right)\right]\right. \\
\left.-2 \sum_{k=0}^{n} \varepsilon_{k} R\left(V_{k}\right)\left(-\lambda\left(N_{k}\right)\right)\right) \varphi(e) \\
=\left(l\left(l-\frac{1}{2}(n+1)\right) \rho L(v(x)) \varphi(e)=l\left(l-\frac{1}{2}(n+1)\right) f(x) .\right.
\end{gathered}
$$

One uses the fact that $\lambda$ acts trivially on $W_{+}$and on $M_{1}$. This proves the first assertion. 
To get the expression for $d \Pi_{\Lambda}\left(\Omega_{L}\right)$ one uses (5.6b) and (4.5). First note that

$$
\sum_{k=0}^{n} \varepsilon_{k} V_{k}^{2}=\frac{1}{4} \sum_{k=0}^{n} \varepsilon_{k} \partial_{k}^{2}=\frac{1}{4} \square .
$$

One computes:

$$
\begin{gathered}
\sum_{k \geq 0}^{n} \varepsilon_{k}\left(\langle x, x\rangle \partial_{k}\right)^{2}=\langle x, x\rangle^{2} \square+2\langle x, x\rangle E, \\
\sum_{k \geq 0}^{n} \varepsilon_{k}\left(x_{k}(E+l \mathbf{1})^{2}\right)=\langle x, x\rangle(E+l \mathbf{1})(E+(l+1) \mathbf{1}), \\
-\sum_{k=0}^{n} \varepsilon_{k}^{2}\left(\langle x, x\rangle \partial_{k} x_{k}(E+l \mathbf{1})+x_{k}(E+l \mathbf{1})\langle x, x\rangle \partial_{k}\right) \\
=-2\langle x, x\rangle(E+l \mathbf{1})\left(E-\frac{1}{2}(n+1) \mathbf{1}\right)-\langle x, x\rangle E,
\end{gathered}
$$

from which one obtains

$$
\sum_{k \geq 0}^{n} \varepsilon_{k} N_{k}^{2}=\frac{1}{4}\langle x, x\rangle^{2} \square+\langle x, x\rangle(E+l \mathbf{1})\left(l-\frac{1}{2}(n-1)\right) .
$$

We also have

$$
\begin{aligned}
-2 \sum_{k=0}^{n} \varepsilon_{k} N_{k} V_{k} & =\frac{1}{2}\langle x, x\rangle \sum_{k=0}^{n} \varepsilon_{k} \partial_{k}^{2}-\sum_{k=0}^{n} \varepsilon_{k}^{2} x_{k}(E+l \mathbf{1}) \partial_{k} \\
& =\frac{1}{2}\langle x, x\rangle \square-(E+l \mathbf{1}) E+E .
\end{aligned}
$$

Noting finally that

$$
d \Pi_{\lambda}\left(H^{2}-\frac{1}{2}(n+1) H\right)=(E+l \mathbf{1})\left(E+(l-1) \mathbf{1}-\frac{1}{2}(n-1) \mathbf{1}\right),
$$

the formula for $d \Pi_{\lambda}\left(\Omega_{L}\right)$ follows. The formula for $d \Pi_{\lambda}\left(\Omega_{K}\right)$ follows from $d \Pi_{\lambda}\left(\Omega_{K}\right)=d \Pi_{\lambda}\left(\Omega_{G}\right)-d \Pi_{\lambda}\left(\Omega_{L}\right)$.

Let $\mathscr{S}_{0}\left(\mathbb{R}^{n+1}\right)$ denote the linear subspace of the Schwartz space $\mathscr{S}\left(\mathbb{R}^{n+1}\right)$ consisting of functions satisfying the wave equation: $\square \Phi=$ 0 . The following observations are important for the following discussion:

Proposition 10. The operator $d \Pi_{\lambda}\left(\Omega_{K}\right)$ commutes with the action $\Pi_{\lambda}$ of the subgroup $\tilde{K}$. If $l=\frac{1}{2}(n-1)$, then the solution set of the wave equation is invariant under the full group $G$.

Proof. By Proposition $7 \Omega_{K}$ is invariant under the subgroup $\tilde{K}$. Since $\Pi_{\lambda}$ is a homomorphism of groups, and $d \Pi_{\lambda}$ is a homomorphism 
of Lie algebras the first result follows easily. To establish the second remark, note first that under the stated condition one has:

$$
d \Pi_{\frac{1}{2}(n-1)}\left(\Omega_{K}\right)=-\frac{1}{4}(1+\langle x, x\rangle)^{2} \square .
$$

It follows from the first remark that this operator commutes with $\Pi_{\lambda}(\tilde{K})$, and hence with the opertors $d \Pi_{\lambda}(\mathbb{G})$ for $\lambda(\exp t H)=e^{\frac{1}{2}(n-1) t}$. In particular, this operator must commute with the operators $d \Pi_{\lambda}\left(X_{i j}\right)$ $=\mathbf{L}_{i j}, 0 \leq i, j \leq n$, as well as with the $d \Pi_{\lambda}\left(X_{k}\right)=\mathbf{N}_{k}-\mathbf{V}_{k}, 1 \leq k \leq n$, and $d \Pi_{\lambda}(Y)=\mathbf{N}_{0}+\mathbf{V}_{0}$. One finds that the following commutators:

$$
\begin{gathered}
{\left[\partial_{j},(1+\langle x, x\rangle)^{2} \square\right]=4 x_{j}(1+\langle x, x\rangle) \square,} \\
{\left[E+l \mathbf{1},(1+\langle x, x\rangle)^{2} \square\right]=2\left(\langle x, x\rangle^{2}+\langle x, x\rangle+1\right) \square .}
\end{gathered}
$$

It follows that if $\Phi \in \mathscr{S}_{0}\left(\mathbb{R}^{n+1}\right)$, then also $d \Pi_{\lambda}\left(V_{j}\right) \Phi=-\partial_{j} \Phi \in$ $\mathscr{S}_{0}\left(\mathbb{R}^{n+1}\right)$, and $d \Pi_{\lambda}(H) \Phi=(E+l 1) \Phi \in \mathscr{S}_{0}\left(\mathbb{R}^{n+1}\right)$. The above remarks show that the space $\mathscr{S}_{0}\left(\mathbb{R}^{n+1}\right)$ is invariant under the operators corresponding to $\mathbb{K}$ as well. However the elements $V_{j}, H$, together with $\mathbb{K}$ span the Lie algebra $G$. Since $G$ is connected, the second result follows.

The following result shows that the eigenspaces for the operator $d \Pi_{\lambda}\left(\Omega_{K}\right)$ can be expressed in terms of solutions of the wave equations for any value of the parameter. This result is of independent interest.

Proposition 11. Let $\Phi$ satisfy the differential equation $d \Pi_{\lambda}\left(\Omega_{K}\right) \Phi$ $=\left[(m+l)^{2}-\frac{1}{2} l(n-1)-m n\right] \Phi$ at its regular points. Let $\Psi=$ $(1+\langle x, x\rangle)^{-m} \Phi$. Then $\Psi$ satisfies the differential equation $\Omega_{K m} \Psi=$ $-(m+l)\left(m+l-\frac{1}{2}(n-1)\right) \Psi$, where

$$
\Omega_{K m}=\frac{1}{4}(1+\langle x, x\rangle) \square+\left(m+l-\frac{1}{2}(n-1)\right) E .
$$

Proof. We have

$$
\begin{aligned}
& d \Pi_{\lambda}\left(\Omega_{K}\right)(1+\langle x, x\rangle)^{m} \Psi \\
& \quad=(1+\langle x, x\rangle)^{m}\left\{d \Pi_{\lambda}\left(\Omega_{K}\right)+\left[d \Pi_{\lambda}\left(\Omega_{K}\right),(1+\langle x, x\rangle)^{m}\right]\right\} \Psi .
\end{aligned}
$$

One computes

$$
\begin{aligned}
& {\left[\square,(1+\langle x, x\rangle)^{m}\right]} \\
& =4(1+\langle x, x\rangle)^{m-2}\left\{(1+\langle x, x\rangle) m\left(m+\frac{1}{2}(n-1)+m E\right)\right. \\
& {\left[\left(l-\frac{1}{2}(n-1)\right) E,(1+\langle x, x\rangle)^{m}\right]} \\
& =m(2 l-n+1)(1+\langle x, x\rangle)^{m} \\
& -m(2 l-n+1)(1+\langle x, x\rangle)^{m-1},
\end{aligned}
$$


so that,

$$
\begin{aligned}
& {\left[d \Pi_{\lambda}\left(\Omega_{K}\right),(1+\langle x, x\rangle)^{m}\right]} \\
& \quad=-(1+\langle x, x\rangle)^{m}\left\{(1+\langle x, x\rangle)\left(m\left(m-\frac{1}{2}(n-1)+E\right)+2 l m\right)\right. \\
& \left.+\left(-m^{2}-2 l m+n m\right) \mathbf{1}\right\} .
\end{aligned}
$$

Thus,

$$
\begin{aligned}
& d \Pi_{\lambda}\left(\Omega_{K}\right) \Phi \\
& \quad=-(1+\langle x, x\rangle)^{m+1}\left\{\frac{1}{4}(1+\langle x, x\rangle) \square+\left(m+l-\frac{1}{2}(n-1)\right) E\right. \\
& \quad+\left((m+l)\left(m+l-\frac{1}{2}(n-1)\right) 1\right\} \Psi \\
& \quad+\left[(m+l)^{2}-\frac{1}{2} l(n-1)-m n\right] \Phi .
\end{aligned}
$$

The result follows.

Now, if in the above result one chooses the parameter $m=$ $\frac{1}{2}(n-1)-l$, then the differential equation for the function $\Psi$ simplifies to

$$
\frac{1}{4}(1+\langle x, x\rangle) \square \Psi=0
$$

Thus one has

Proposition 12. Set $\Psi=(1+\langle x, x\rangle)^{\frac{1}{2}(n-1)-l} \Phi$. Then on the regular points, $\Phi$ satisfies the equation

$$
d \Pi_{\lambda}\left(\Omega_{K}\right) \Phi=\frac{1}{2}(n+1)\left[l-\frac{1}{2}(n-1)\right] \Phi,
$$

if and only if $\Psi$ satisfies the wave equation $\square \Psi=0$.

7. Representation defined by an integral transform of the solution space of the wave equation. Define the space $\mathscr{S}^{2}\left(\mathbb{R}^{n}\right)$ to be the linear space of rapidly decreasing $C^{\infty}$-functions $\Phi$ for which the integrals

$$
\int_{\mathbb{R}^{n}} \Phi(\mathbf{y}) \operatorname{conj}[\Phi(\mathbf{y})] \frac{d^{n} y}{r(\mathbf{y})}
$$

converge. We write $r=r(\mathbf{y})=\sqrt{(\mathbf{y}, \mathbf{y})}$, where $(\mathbf{y}, \mathbf{x})$ for $\mathbf{x}, \mathbf{y} \in \mathbb{R}^{n}$ is the usual inner product on $\mathbb{R}^{n}$. We also write for $x \in \mathbb{R}^{n+1}, x=\left(x_{0}, \mathbf{x}\right)$, with $\mathbf{x} \in \mathbb{R}^{n}, x_{0} \in \mathbb{R}$.

Let $\mathscr{E}$ denote the function $\mathscr{E}: \mathbb{R}^{n+1} \times \mathbb{R}^{n} \Rightarrow \mathbb{C}$ defined by $\mathscr{E}(x, \mathbf{y})=$ $\exp i\left\{(\mathbf{x}, \mathbf{y})+x_{0} r(\mathbf{y})\right\}$, where as before $i=\sqrt{-1}$. Define the transform $S: \mathscr{S}^{2}\left(\mathbb{R}^{n}\right) \Rightarrow C^{\infty}\left(\mathbb{R}^{n+1}\right)$ by

$$
(S \Phi)(x)=\int_{\mathbb{R}^{n}} \mathscr{E}(x, \mathbf{y}) \Phi(\mathbf{y}) \frac{d^{n} y}{r(\mathbf{y})},
$$


$x \in \mathbb{R}^{n+1}, \Phi \in \mathscr{S}^{2}\left(\mathbb{R}^{n}\right)$. One checks easily that the functions $x \Rightarrow$ $\mathscr{E}(x, y)$ satisfy the wave equation. It follows then from a uniform continuity argument that the image of $S$ consists of solutions of the wave equation.

We compute now the transfer of the infinitesimal action of the Lie algebra $\mathbf{G}=\operatorname{so}(2, n+1)$ given in Proposition 8 or in (4.5) to the space $\mathscr{S}^{2}\left(\mathbb{R}^{n}\right)$. Let $\Delta$ and $F$ denote the Laplacian and the symmetrized Euler operator on $\mathbb{R}^{n}$ as defined in $\S 2$. We also write $E_{x}=E$ and $E=E_{y}$ for the Euler operator on $\mathbb{R}^{n+1}$, and $\mathbb{R}^{n}$ respectively. When it is necessary to call attention to the coordinate variables the subscripts will be employed. One has the following result:

THEOREM 3. The image of $S$ is invariant under the infinitesimal action of so $(2, n+1)$ defined by (1), and this action is transferred to $\mathscr{S}^{2}\left(\mathbb{R}^{n}\right)$ as follows. For the infinitesimal translations:

$$
V_{j} S=-i S \frac{1}{2} y_{j}, \quad V_{0} S=-i S \frac{1}{2} r(\mathbf{y}) .
$$

For the elements of $\mathrm{M}_{1}$ :

$$
\begin{aligned}
& \left(x_{j} \frac{\partial}{\partial x_{0}}+x_{0} \frac{\partial}{\partial x_{j}}\right) S=-S r \frac{\partial}{\partial y_{j}}, \\
& \left(x_{j} \frac{\partial}{\partial x_{k}}-x_{k} \frac{\partial}{\partial x_{j}}\right) S=S\left(y_{j} \frac{\partial}{\partial y_{k}}-y_{k} \frac{\partial}{\partial y_{j}}\right) .
\end{aligned}
$$

For the infinitesimal scale changes one has:

$$
\left(E+\frac{1}{2}(n-1) 1\right) S=-S\left(\mathbb{F}_{y}-\frac{1}{2} \mathbf{1}\right) .
$$

For the infinitesimal conjugate translations:

$$
N_{j} \mathbb{S}=\mathbb{S}-i\left\{\frac{1}{2} \Delta y_{j}-\frac{\partial}{\partial y_{j}}\left(F-\frac{1}{2} \mathbf{1}\right)\right\},
$$

$1 \leq j \leq n$.

$$
N_{0} S=-S i \frac{1}{2} r(\mathbf{y}) \Delta .
$$

Proof. Let $(,)_{j}$ denote the duality $\mathscr{S}\left(\mathbb{R}^{n}\right) \times \mathscr{S}^{\prime}\left(\mathbb{R}^{n}\right) \Rightarrow \mathbb{C}$ defined by

$$
(\phi, \psi)_{y}=\int_{\mathbb{R}^{n}} \phi(\mathbf{y}) \bar{\psi}(\mathbf{y}) d y,
$$

$\varphi \in \mathscr{S}\left(\mathbb{R}^{n}\right), \psi \in \mathscr{S}^{\prime}\left(\mathbb{R}^{n}\right)$. In this notation the definition of the transform (7.2) can be written:

$$
(S \varphi)(x)=(\mathscr{E}(x, \cdot),(1 / r) \bar{\varphi})_{y} .
$$


For a differential operator $T$ define the operator $T^{*}$ on $\mathscr{S}\left(\mathbb{R}^{n}\right)$ by $\left(T^{*} \varphi, \psi\right)_{y}=(\varphi, T \psi)_{y}, \varphi \in \mathscr{S}\left(\mathbb{R}^{n}\right), \psi \in \mathscr{S}^{\prime}\left(\mathbb{R}^{n}\right)$. Clearly one has $(A B)^{*}=B^{*} A^{*}$, for differential operators $A$, and $B$. A differential operator $T$ is called formally self adjoint if $T^{*}=T$. If $T$ is formally self adjoint then $(i T)^{*}=-i T$. It is clear that the operators of multiplication by real-valued functions are formally self adjoint. It is also a fact that the operators $\Delta_{y}$ and $i F_{y}$ are formally self adjoint. This remark follows easily by integration by parts. By integration by parts one also deduces that the operators $i \partial / \partial y_{j}$ for $1 \leq j \leq n$ are formally self adjoint. If $U$ is a differential operator with complex coefficients, $\overline{\mathbf{U}}$ denotes the operator whose coefficients are the complex conjugates of those of $U$.

First for the infinitesimal translations $\mathbf{V}_{i}$ note that:

$$
\begin{gathered}
V_{j} \mathscr{E}(x, \mathbf{y})=-\frac{1}{2} \partial / \partial x_{j} \mathscr{E}(x, \mathbf{y})=-i y_{j} \mathscr{E}(x, \mathbf{y}), \quad 1 \leq j \leq n \\
V_{0} \mathscr{E}(x, \mathbf{y})=-\frac{1}{2} \partial / \partial x_{0} \mathscr{E}(x, \mathbf{y})=-i \frac{1}{2} r(\mathbf{y}) \mathscr{E}(x, \mathbf{y})
\end{gathered}
$$

Then from the above considerations and (7.3),

$$
\begin{aligned}
\left(V_{j} S \varphi\right)(x) & =\frac{1}{2}\left(-i y_{j} \mathscr{E}(x, \mathbf{y}),(1 / r) \bar{\phi}\right) y \\
& =\frac{1}{2}\left(\mathscr{E}(x, \mathbf{y}),(1 / r) \overline{-i y_{j}} \phi\right)=-\frac{1}{2}\left(\operatorname{Siy}_{j} \varphi\right)(x)
\end{aligned}
$$

Similarly from (7.4),

$$
\left(V_{0} S \varphi\right)(x)=-\frac{1}{2}(\operatorname{Sir} \varphi(x) .
$$

This establishes the first line in the theorem.

In order to establish some of the remaining formulae, note that the definition of formal adjoint implies

$$
(T \mathscr{E}(x, \cdot),(1 / r) \bar{\varphi})_{y}=\left(S\left(\overline{\mathbf{T}}^{*}+\left[\overline{\mathbf{T}}^{*}, 1 / r\right]\right) \varphi\right)(x) .
$$

Then for the operators corresponding to the elements of the subalgebra $M_{1}$, we note that

$$
\begin{aligned}
r(\mathbf{y}) \frac{\partial}{\partial y_{j}} \mathscr{E}(x, \mathbf{y}) & =i\left(x_{j} r(\mathbf{y})+x_{0} y_{j}\right) \\
& =\left(x_{j} \frac{\partial}{\partial x_{0}}+x_{0} \frac{\partial}{\partial x_{j}}\right) \mathscr{E}(x, \mathbf{y}) .
\end{aligned}
$$


Then for $\varphi \in \mathscr{S}^{2}\left(\mathbb{R}^{n}\right)$,

$$
\begin{aligned}
\left(x_{j} \frac{\partial}{\partial x_{0}}+x_{0} \frac{\partial}{\partial x_{j}}\right)(S \varphi)(x) & =\left(r \frac{\partial}{\partial y_{j}} \mathscr{E}(x, \cdot), \frac{1}{r} \varphi\right) \\
& =-\left(\mathscr{E}(x, \cdot), \frac{\partial}{\partial y_{j}} \varphi\right) \\
& =-\left(\mathscr{E}(x, \cdot),(1 / r) r \frac{\partial \phi}{\partial y_{j}}\right) .
\end{aligned}
$$

Formula (ii) follows. For $1 \leq j<k \leq n$,

$$
\begin{aligned}
\left(y_{j} \frac{\partial}{\partial y_{k}}-y_{k} \frac{\partial}{\partial y_{j}}\right) \mathscr{E}(x, \mathbf{y}) & =i\left(y_{j} x_{k}-y_{k} x_{j}\right) \mathscr{E}(x, \mathbf{y}) \\
& =\left(x_{k} \frac{\partial}{\partial x_{j}}-x_{j} \frac{\partial}{\partial x_{k}}\right) \mathscr{E}(x, \mathbf{y}) .
\end{aligned}
$$

One uses (7.5) noting that if $T$ is an infinitesimal rotation on $\mathbb{R}^{n}$, then $T^{*}=-T$, and $T$ commutes with functions of $r$. Formula (7.7) then implies (iii). To work out the action of $H$,

$$
E_{\mathbf{y}} \mathscr{E}(x, \mathbf{y})=i\left[(\mathbf{x}, \mathbf{y})+x_{0} r(\mathbf{y})\right] e(x, \mathbf{y})=E_{x} \mathscr{E}(x, \mathbf{y}) .
$$

Again one uses (7.5), noting that $i F_{y}$ is formally self adjoint, and $\{F, 1 / r]=-1 / r$. Formula (iv) follows easily from these observations.

Next, a straightforward computation shows that

$$
\begin{aligned}
\Delta_{y} \mathscr{E}(x, \mathbf{y})= & -\left(\mathbf{x}+\frac{x_{0} \mathbf{y}}{r(\mathbf{y})}, \mathbf{x}+\frac{x_{0} \mathbf{y}}{r(\mathbf{y})}\right) \mathscr{E}(x, \mathbf{y}) \\
& +i(n-1) \frac{x_{0}}{r(\mathbf{y})} \mathscr{E}(x, \mathbf{y}) .
\end{aligned}
$$

Note that $\Delta_{y} y_{j}=y_{j} \Delta_{y}+2 \partial / \partial y_{j}$, thus,

(7.10) $y_{j} \Delta_{y} \mathscr{E}(x, \mathbf{y})$

$$
\begin{aligned}
= & \left(-y_{j}\left(\mathbf{x}+\frac{x_{0} \mathbf{y}}{r(\mathbf{y})}, \mathbf{x}+\frac{x_{0} \mathbf{y}}{r(\mathbf{y})}\right)+\frac{y_{j} x_{0}(n-1)}{r(\mathbf{y})}\right) \mathscr{E}(x, \mathbf{y}) \\
= & \left\{-y_{j}\left((\mathbf{x}, \mathbf{x})+x_{0}^{2}+\frac{2(\mathbf{x}, \mathbf{y}) x_{0}}{r(\mathbf{y})}\right)\right. \\
& \left.+\frac{i(n-1) y_{j} x_{0}}{r(\mathbf{y})}\right\} \mathscr{E}(x, \mathbf{y}) .
\end{aligned}
$$


From (7.8) one has for $1 \leq j \leq n$,

$$
\begin{aligned}
\frac{\partial}{\partial y_{j}}( & \left.E+\frac{1}{2}(n-3) \mathbf{1}\right) \mathscr{E}(x, \mathbf{y}) \\
=\left\{i \frac{1}{2}(n-1)\left(x_{j}+\frac{x_{0} y_{j}}{r(\mathbf{y})}\right)-(\mathbf{x}, \mathbf{y})\right. & \left(x_{j}+\frac{x_{0} y_{j}}{r(\mathbf{y})}\right) \\
& \left.-x_{0} x_{j} r(\mathbf{y})-x_{0}^{2} y_{j}\right\} .
\end{aligned}
$$

Thus, using $\langle x, x\rangle=-x_{0}^{2}+(\mathbf{x}, \mathbf{x})$,

$$
\begin{aligned}
\left(\frac{1}{2} y_{j} \Delta\right. & \left.-\frac{\partial}{\partial y_{j}}\left(E+\frac{1}{2}(n-3) \mathbf{1}\right)\right) \mathscr{E}(x, \mathbf{y}) \\
= & -i\left\{-\frac{1}{2}\langle x, x\rangle \frac{\partial}{\partial x_{j}}+x_{j}\left(E_{x}+\frac{1}{2}(n-1) \mathbf{1}\right)\right) \mathscr{E}(x, \mathbf{y}) \\
= & i N_{j} \mathscr{E}(x, \mathbf{y})
\end{aligned}
$$

by (4.5). To prove (v) one uses (7.5). Here one sets

$$
T=i\left(\frac{1}{2} y_{j} \Delta-\frac{\partial}{\partial y_{j}}\left(E+\frac{1}{2}(n-3)\right)\right) .
$$

Then $\overline{\mathbf{T}}^{*}=-T^{*}$, and

$$
\mathbf{T}^{*}=-i\left(\frac{1}{2} \Delta y_{j}-\left(F+\frac{3}{2}\right) \frac{\partial}{\partial y_{j}}\right) .
$$

A straightforward computation shows that $\left[T^{*}, 1 / r\right]=-i(1 / r) \partial / \partial y_{j}$. Note that $\left(F+\frac{1}{2} \mathbf{1}\right) \partial / \partial y_{k}=\partial / \partial y_{k}\left(F-\frac{1}{2} \mathbf{1}\right)$. Formula (v) follows.

Finally from (7.9) one has

$$
\begin{aligned}
& r(\mathbf{y}) \Delta_{y} \mathscr{E}(x, \mathbf{y}) \\
& \quad=\left\{i x_{0}(n-1)-r(\mathbf{y})(\mathbf{x}, \mathbf{x})-x_{0}^{2} r(\mathbf{y})-2 x_{0}(\mathbf{x}, \mathbf{y})\right\} \mathscr{E}(x, \mathbf{y}) .
\end{aligned}
$$

From (7.8) then,

(7.11) $r(\mathbf{y}) \Delta_{y} \mathscr{E}(x, \mathbf{y})$

$$
\begin{aligned}
& =-i 2\left\{-\frac{1}{2}\langle x, x\rangle \partial / \partial x_{0}-x_{0}\left(E_{x}+\frac{1}{2}(n-1) \mathbf{1}\right\} \mathscr{E}(x, \mathbf{y})\right. \\
& =2 i N_{0} \mathscr{E}(x, \mathbf{y}),
\end{aligned}
$$

by (4.5). Therefore for $\psi \in \mathscr{S}^{2}\left(\mathbb{R}^{n}\right)$, one has from (7.2) and (7.5)

$$
\begin{aligned}
\mathbf{N}_{0}(S \psi)(x) & =-\frac{1}{2}(\operatorname{ir} \Delta \mathscr{E}(x, \cdot),(1 / r) \bar{\psi})_{y}=\frac{1}{2}(\mathscr{E}(x, \cdot), i \Delta \bar{\psi})_{y} \\
& =-\frac{1}{2} S(i r \Delta \psi)(x) .
\end{aligned}
$$

This establishes (vi). 
It is now possible to give the alternative discussion of Theorem 1 mentioned in $\S 2$. The formal verification of the table in Theorem 1 is immediate from the last proposition. It is necessary to make precise the space on which these operators act. We know that the operators given by (4.5) span a real Lie algebra, because by Proposition 8 they are the differentials of a group action of the group $\mathrm{O}(2, n+1)$ on the space $\mathscr{S}\left(\mathbb{R}^{n+1}\right)$. Moreover for the case considered here the parameter $l=\frac{1}{2}(n-1)$. Then we know from Proposition 10 the set of solutions of the wave equation defines an invariant linear subspace of $\mathscr{S}\left(\mathbb{R}^{n+1}\right)$. Now consider the function $\varphi=\exp (-r(\mathbf{y})), \mathbf{y} \in \mathbb{R}^{n}$. Clearly this function is in the space $\mathscr{S}^{2}\left(\mathbb{R}^{n}\right)$. Hence, it is mapped by the transform $S$ into the solution space of the wave equation. Let $\mathscr{S}_{0}\left(\mathbb{R}^{n+1}\right)$ be the subspace of this solution space spanned by the group translates $\Pi_{\lambda}(g) S e^{-r(\cdot)}, g \in G$. By Proposition 10 again, this subspace is contained in the solution space of the wave equation.

Let $\left(\right.$, ) be the inner product on $\mathscr{S}^{2}\left(\mathbb{R}^{n}\right)$ defined by

$$
(\Phi, \Psi)=\int_{\mathbb{R}^{n}} \Phi(\mathbf{y}) \operatorname{conj}[\Psi(\mathbf{y})] \frac{d^{n} y}{r(\mathbf{y})} .
$$

The definition of the space $\mathscr{S}^{2}\left(\mathbb{R}^{n}\right)$ and the Cauchy-Schwarz inequality implies that the integrals in (7.12) exist. Hence this is an inner product on $\mathscr{S}^{2}\left(\mathbb{R}^{n}\right)$.

Next note that the transform $S$ is one to one. This remark follows from Definition 7.2 and the fact that the Fourier transform on $\mathbb{R}^{n}$ is one to one on the Schwartz space. This is an immediate consequence of the Plancherel theorem. Hence $S \varphi=0$ implies $\exp \left(i x_{0} r(\mathbf{y}) \varphi / r\right)=$ 0 . Thus $\varphi=0$. This proves the remark.

Since the transform $S$ is one to one the preimage of $\mathscr{S}_{0}\left(\mathbb{R}^{n+1}\right)$ is a well defined linear subspace of $\mathscr{S}^{2}\left(\mathbb{R}^{n}\right)$, which we denote by $\mathscr{S}_{0}^{2}\left(\mathbb{R}^{n}\right)$. One can and does also define an inner product on $\mathscr{S}_{0}\left(\mathbb{R}^{n+1}\right)$

$$
\langle S \Phi, S \Psi\rangle=(\Phi, \Psi),
$$

$\Phi, \Psi \in \mathscr{S}_{0}^{2}\left(\mathbb{R}^{n}\right)$. Thus the action of $G$ on $\mathscr{S}_{0}\left(\mathbb{R}^{n+1}\right)$ is transferred to an action of the group $G$ on $\mathscr{S}_{0}^{2}\left(\mathbb{R}^{n}\right)$. It is claimed that this action is preunitary with respect to the inner product defined in (7.13). It will follow from this last assertion that there is a uniquely defined unitary representation the completion of $\mathscr{S}_{0}^{2}\left(\mathbb{R}^{n}\right)$ with respect to the metric defined in (7.12). The claim now follows from the following considerations: Let $S^{-1}$ denote the inverse of $S$ on the range of the latter transform. Then by definition the action of the group $G$ on the space $\mathscr{S}_{0}^{2}\left(\mathbb{R}^{n}\right)$ is given by the operators $S^{-1} \pi_{\lambda}(g) S$ for $g \in G$. The 
infinitesimal generators of the one parameter subgroups of this action are elements of the real linear span of the operators list on the right in the table of Theorem 1. Thus it is sufficient to check that these operators are formally skew-adjoint with respect to the inner product defined in (7.12). For all except for the next to last member of the list this result is a fairly straightforward consequence of the facts that the operators $i r, i \Delta, L_{i j} \partial_{k}$ are formally skew adjoint with respect to the usual $L_{2}$-inner product on $L_{2}\left(\mathbb{R}^{n}\right)$. The details are left to the reader. The problematic verification is to check the formal skew-adjointness of the next to the last operators $i\left(\partial_{k}\left(F-\frac{1}{2} \mathbf{1}\right)-\frac{1}{2} \Delta y_{k}\right)$. For this purpose, let $(,)_{2}$ denote the $L^{2}$ inner product. It is defined on $\mathscr{S}_{0}^{2}\left(\mathbb{R}^{n}\right)$. Then for $\varphi, \psi \in \mathscr{S}_{0}^{2}\left(\mathbb{R}^{n}\right)$, one has

$$
\begin{aligned}
\left(i\left(\partial_{k}\left(F-\frac{1}{2} \mathbf{1}\right)-\frac{1}{2} \Delta y_{k}\right) \varphi, \psi\right)=\left(i\left(\partial_{k}\left(F-\frac{1}{2} \mathbf{1}\right)-\frac{1}{2} \Delta y_{k}\right) \varphi, \psi / r\right)_{2} \\
=-\left(\varphi, i\left(\left(F+\frac{1}{2} \mathbf{1}\right) \partial_{k}-\frac{1}{2} y_{k} \Delta\right) \psi / r\right)_{2} \\
=\left(\varphi,\left\{i\left(\left(F+\frac{1}{2} \mathbf{1}\right) \partial_{k}-\frac{1}{2} y_{k} \Delta\right)\right.\right. \\
\left.\left.+\quad+r\left[i\left(\left(F+\frac{1}{2} \mathbf{1}\right) \partial_{k}-\frac{1}{2} y_{k} \Delta\right), 1 / r\right]\right\} \psi\right) .
\end{aligned}
$$

One notes that $\left[\left(F+\frac{1}{2} \mathbf{1}\right), \partial_{k}\right]=-\partial_{k}$, and $\left[y_{k}, \Delta\right]=-2 \partial_{k}$. Thus one has

$$
\left(F+\frac{1}{2} \mathbf{1}\right) \partial_{k}-\frac{1}{2} y_{k} \Delta=\partial_{k}\left(F+\frac{1}{2} \mathbf{1}\right)-\frac{1}{2} \Delta y_{k} .
$$

The commutator is computed as follows:

$$
\begin{aligned}
{[(F} & \left.\left.+\frac{1}{2} \mathbf{1}\right) \partial_{k}-\frac{1}{2} x_{k} \Delta, \frac{1}{r}\right] \\
& =-\frac{1}{r} \partial_{k}+\left(F+\frac{1}{2} \mathbf{1}\right)\left(-\frac{x_{k}}{r^{3}}\right)-\frac{1}{2} x_{k}\left[\Delta, \frac{1}{r}\right] \\
& =-\frac{1}{r} \partial_{k}+\left(-\frac{x_{k}}{r}\right)\left(F-\frac{3}{2} \mathbf{1}\right)-\frac{1}{2} x_{k}\left[\Delta, \frac{1}{r}\right] .
\end{aligned}
$$

For the last term one finds

$$
\left[\Delta, \frac{1}{r}\right]=-\frac{1}{r^{3}}(F-3) \text {. }
$$

It follows that

$$
\left[\left(F+\frac{1}{2} \mathbf{1}\right) \partial_{k}-\frac{1}{2} x_{k} \Delta, \frac{1}{r}\right]=-\frac{1}{r} \partial_{k} .
$$

Thus from (7.14)

$$
\left(i\left(\partial_{k}\left(F-\frac{1}{2} \mathbf{1}\right)-\frac{1}{2} \Delta y_{k}\right) \varphi, \psi\right)=-\left(\varphi,\left(\partial_{k}\left(F-\frac{1}{2} \mathbf{1}\right)-\frac{1}{2} \Delta y_{k}\right) \psi\right) .
$$

The next theorem follows immediately from these considerations. 
THEOREM 4. For each positive integer $n \geq 1$ there exists a unitary representation of the groups $G$. These representations are defined on the completions of the spaces $\mathscr{S}_{0}\left(\mathbb{R}^{n+1}\right)$ with respect to the Hilbert space metric defined by (4.13).

In the next paper of this series we shall prove the irreducibility of these representations. It will also follow from those considerations that these Hilbert spaces are the completions of the entire images of the spaces $\mathscr{S}^{2}\left(\mathbb{R}^{n}\right)$ under the transform $S$.

\section{REFERENCES}

[A1] L. V. Ahlfors, Möbius Transformations In Several Dimensions, Dept. of Math. University of Minnesota Lecture Notes (1981).

[A2] _ Möbius Transformations and Clifford Numbers in Differential Geometry and Complex Analysis, I. Chavel, H. M. Farkas (Eds.) Springer Verlag Berlin Heidelberg (1985).

[A] S. P. Alliluev, On the relation between "accidental" degeneracy and "hidden symmetry of a system, J. Exptl. Theor. Phys. (U.S.S.R.), 33 (1957), 200-203. Trans: 6, (1958), 156-159.

[B1] V. Bargmann, Zur Theorie des Wasserstoffatoms. Bemerkungen zur gleichamigen Arbeit von V. Fock, Z. Phys., 99 (1936), 576-581.

[Ba1] A. O. Barut, Dynamical Groups and Generalized Symmetries in Quantum Theory, University of Canterbury, Christchurch, New Zealand (1972).

[Ba2] _ Dynamical Groups for the Motion of Relativistic Composite Systems, in Groups Systems and Many-Body Physics, Kramer and Cin ed. (1980).

[E] J. Elhadad, Sur l'interprétation en géométrie symplectique des états quantiques de l'atome d'hydrogène, Symposia Mathematica, Instituto Nazionale D1 Alta Matematica XIV Academic Press, (1974), 259-291.

[F] V. Fock, Zur Theorie der Wasserstoffatoms, Z. Phys., 98 (1935), 145-154.

[He] S. Helgason, Differential Geometry, Lie Groups, and Symmetric Spaces, Academic Press (1978).

[Se1] I. E. Segal, Mathematical Problems of Relativistic Physics, Amer. Math. Soc., (1963).

[Se2] A class of operator algebras which are determined by groups, Duke Math. J., 18 (1951), 221-265.

[So1] J. M. Souriau, Sur La Variété de Képler Symposia Mathematica, Instituto Nazionale Di Alta Matematica XIV Academic Press, (1974).

[So2] _ Structure des Systèmes Dynamiques, Dunod, Paris, (1970).

[Sw] L. Schwartz, Théorie des Distributions, Hermann, Paris, (1966).

[Th] E. Thieleker, Some results on the pseudo-orthogonal groups and their spinor groups, to appear.

Received October 27, 1987.

UNIVERSITY OF SOUTH FLORIDA

TAMPA, FL 33620 